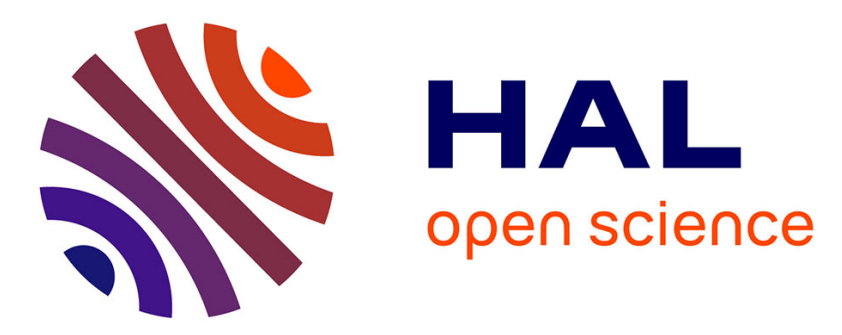

\title{
Influence Of Bottom Topography On An Upwelling Current: Generation Of Long Trapped Filaments
}

\author{
T. Meunier, V. Rossi, Y. Morel, X. Carton
}

\section{To cite this version:}

T. Meunier, V. Rossi, Y. Morel, X. Carton. Influence Of Bottom Topography On An Upwelling Current: Generation Of Long Trapped Filaments. Ocean Modelling, 2010, 35 (4), pp.277-303. 10.1016/j.ocemod.2010.08.004 . hal-00998661

\section{HAL Id: hal-00998661 https://hal.science/hal-00998661}

Submitted on 4 Jun 2014

HAL is a multi-disciplinary open access archive for the deposit and dissemination of scientific research documents, whether they are published or not. The documents may come from teaching and research institutions in France or abroad, or from public or private research centers.
L'archive ouverte pluridisciplinaire HAL, est destinée au dépôt et à la diffusion de documents scientifiques de niveau recherche, publiés ou non, émanant des établissements d'enseignement et de recherche français ou étrangers, des laboratoires publics ou privés. 


\title{
Influence of bottom topography on an upwelling current : generation of long trapped filaments
}

\author{
T. Meunier,a, V. Rossi ${ }^{\mathrm{b}}$, Y. Morel ${ }^{\mathrm{c}}, \mathrm{X}$. Carton $^{\mathrm{a}}$ \\ ${ }^{a}$ Laboratoire de Physique de l'Océan, Université de Bretagne Occidentale, 46 avenue Legorgeu, 29200 Brest, France \\ ${ }^{b}$ Laboratoire d'Études en Géophysique et Océanographie Spatiale, CNRS, Observatoire Midi-Pyrénées, 14 avenue \\ Edouard Belin, 31400 Toulouse, France \\ ${ }^{c}$ Service hydrographique et océanographique de la marine, (SHOM), 42 av Gaspard Coriolis, 31057 Toulouse, France
}

\begin{abstract}
We investigate the influence of bottom topography on the formation and trapping of long upwelling filaments. They use a 2-layer shallow water model on the f-plane. A wind forced alongshore current, associated with coastal upwelling along a vertical wall, encounters a promontory of finite width and length, perpendicular to the coast.

In the lower layer, topographic eddies form, which are shown to drive the formation of a filament on the front. Indeed, as the upwelling current and front develop along the coast, the along shore flow crosses the promontory, re-arranging the potential vorticity structure and generating intense vortical structures : water columns with high potential vorticity initially localized upon the promontory are advected into the deep ocean, forming cyclonic eddies, while water columns from the deep ocean with low potential vorticity climb on the topography forming a trapped anticyclonic circulation. These topographic eddies interact with the upper layer upwelling front and form an elongated, trapped and narrow filament.
\end{abstract}

Sensitivity tests are then carried out and it is shown that :

- baroclinic instability of the front does not play a major role on the formation of long trapped filaments;

- increasing the duration of the wind forcing increases the upwelling current and limits the offshore growth of the filament;

- modifying the promontory characteristics (width, length, height and slopes) has strong impact on the filament evolution, sometimes leading to a multipolarisation of the potential vorticity anomaly structure which results in much more complicated patterns in the upper layer (numerous shorter and less coherent filaments). This shows that only specific promontory shapes can lead to the formation of well defined filaments;

- adding bottom friction introduces a slight generation of potential vorticity in the bottom layer over the promontory, but does not significantly alter significantly the formation of the filament along the outcropped front in the present configuration;

- modifying the stratification characteristics, in particular the density jump between the layers, has only a weak influence on the dynamics of topographic eddies and on filament formation; 
- the influence of capes is also modest in our simulations, showing that topography plays the major role in the formation of long and trapped upwelling filaments.

Key words: Eastern boundary, Mesoscale dynamics, Upwelling/Downwelling, Topographic flows, Upwelling filaments, Potential vorticity

\section{Introduction}

Long trapped filaments of cold water are ubiquitous features along upwelling fronts. They sometimes extend hundreds of kilometers offshore and have been shown to play a major role in the offshore transport of recently upwelled coastal water ([Kostianoy and Zatsepin, 1996]) and in feeding the oligotrophic offshore waters with nutrients and organic materials ([Alvarez-Salgado et al., 2007] and more particularly over the Iberian peninsula upwelling [Alvarez-Salgado et al., 2001]). They are thus important physical features for eastern boundary upwelling ecosystems.

Figure 1 shows a set of satellite images of the Iberian peninsula on the 09/05/2009 (column 1) and 09/05/2005 (column 2).. The images on the first row are the Sea Surface Temperature (SST) and on the second row the Chlorophyll-a concentration (Sea Surface Color). Long, trapped and recurrent filaments are observed on SST and chlorophyll maps off Cape Finisterre $\left(43^{\circ} \mathrm{N}\right)$, São Vicente $\left(37^{\circ} \mathrm{N}\right)$ and the Estremadura promontory (between 38.5 and $\left.39.5^{\circ} \mathrm{N}\right)$. However on all maps, another tongue of cold upwelled water pointing offshore also clearly arises from the upwelling front just South of the Estremadura promontory.

In-situ and remotely sensed data have provided some desriptions of upwelling filaments (see for instance [Brink, 1983]; [Flament et al., 1985]; [Washburn and Armi, 1988]; [Strub et al., 1991]; [Navarro-Pérrez and Barton, 1998]; [Barton, 2001]), and many numerical and theoretical studies have focused on their dynamics. [Haynes et al., 1993] used satellite data of the West-Iberia upwelling system to show that large filaments were often closely related with capes, but noted the repeated occurrence of two large filaments at two different locations corresponding with a straight coastline. They assumed that those filaments were related with unstable meanders of the jet, but their stationarity remained unexplained.

One of the first process studies on upwelling filament dynamics was performed by [Haidvogel et al., 1991]. Their model was able to reproduce cold filaments along the upwelling front. They concluded from a set of sensitivity cases, including removal of the coastline geometry and the bottom topography, that the presence of large capes along the coast, was necessary to the generation of upwelling filaments. [Marchesiello et al., 2003] studied the equilibrium structure of the California current system and conducted different sensitivity tests. They showed that mesoscale variability was intrinsic to the current and not due to the variability of the forcing. Contrary to [Haidvogel et al., 1991], they showed that even in the case of a straight coastline and a flat bottom, upwelling filaments and eddies still occurred, but with no preferential location, confirming the conclusions of [Roed and Shi, 1999], that instability induced filaments and eddies could happen in the absence of coastal irregularities. Removing only the topography,

\footnotetext{
${ }^{*}$ Corresponding author

Email addresses: thomas.meunier@univ-brest.fr (T. Meunier), vincent.rossi@legos.obs-mip.fr (V. Rossi), yves.morel@shom.fr (Y. Morel), xavier.carton@univ-brest.fr (X. Carton) 

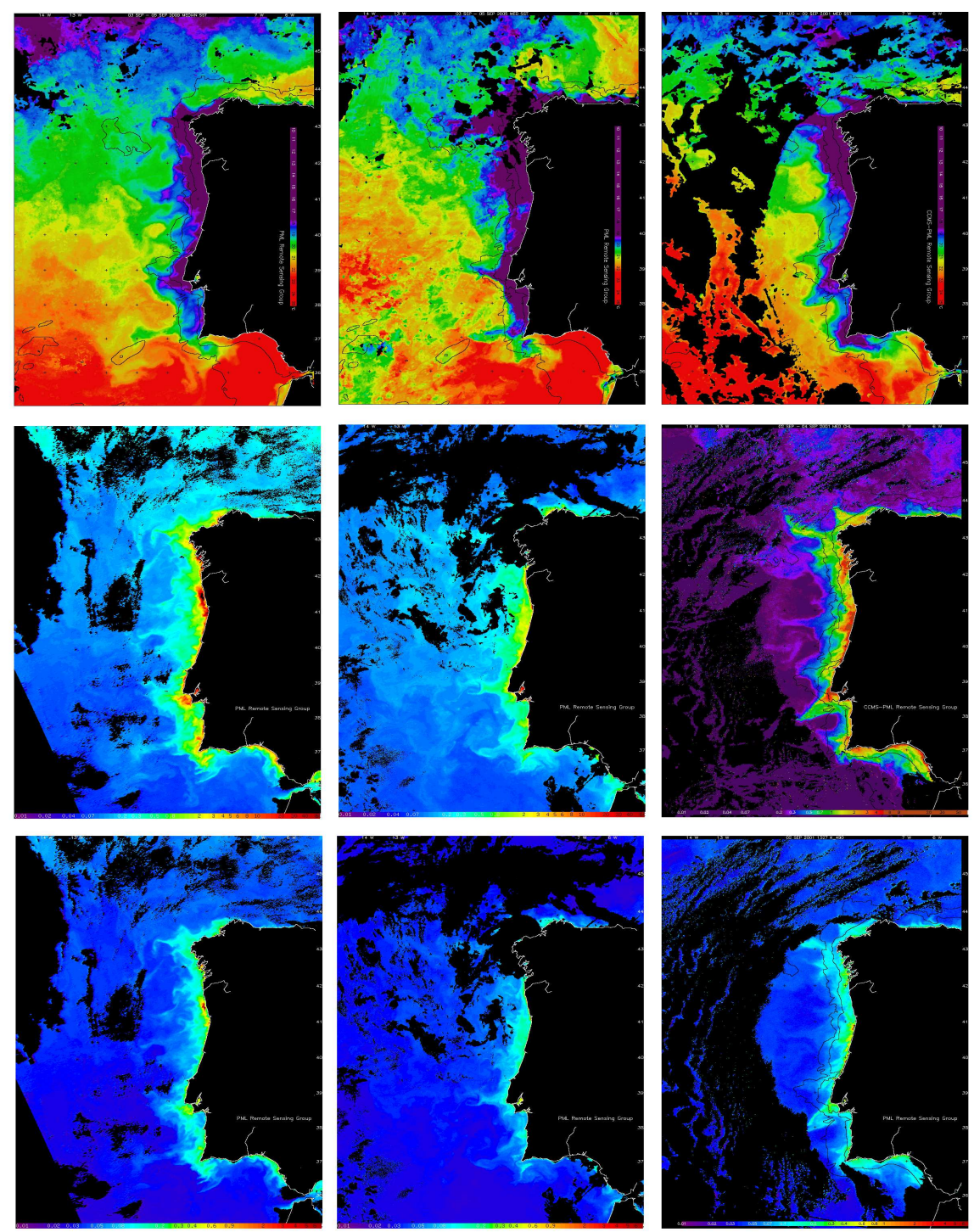

Figure 1: Sea Surface Temperature (first column) and Chlorophyll distribution (second column) situation off the West Iberian coast during three distinct upwelling episodes (04/09/2005 on the first row and 05/09/2009 on the second row.) 
[Marchesiello et al., 2003] concluded that a standing wave could be generated at the cape, in agreement with results from a numerical study of [Batteen, 1997] who noted anchorement of filaments at coastline irregularities, while [Roed and Shi, 1999] only noted a weak anchoring of the meander. [Ikeda, 1981] and [Ikeda, 1989] showed using two-layer quasigeostrophic models, that unstable meanders of a buoyancy driven coastal jet would move downstream by a combined effect of propagation and advection, so that some physical process had to be involved in the anchoring of filaments at the capes as observed by [Marchesiello et al., 2003].

Recently [Batteen et al., 2007] conducted a process oriented study of the Northern Canary Current System (NCCS) and revisited the upwelling instability and filamentation problem in this region. They modified the bottom topography, boundary conditions and wind forcing and found that :

- the flat bottom experiment shows many of the typically observed features of the NCCS;

- adding the bottom topography shows that topography has an important role in intensifying and trapping the equatorward current near the coast, in weakening the subsurface poleward current, and in intensifying eddies off the capes of Iberia;

- the flat bottom experiment produces anticyclonic meanders near cabo Roca and Cabo São Vincente, but not off Figueira da Foz;

- the beta effect plays an important role on the formation of the subsurface meander off cabo Roca.

To summarize, four main source have been identified to explain the generation of filaments along upwelling fronts :

- the frontal or baroclinic instability of the front;

- the effect of capes;

- the planetary beta effect;

- bottom topography.

Upwelling filaments have also been classified into different types (see [Strub et al., 1991]), but one important distinction is whether they are trapped or not. Because the trapping of filaments always happens at the same locations, we believe that the observed long trapped upwelling filaments are associated with topographic features and we chose to focus on this aspect in the present study. Again, different studies ([Ikeda, 1989, Capet and Carton, 2004]) concluded that topographic irregularities were destabilization source for upwelling fronts, but the effect of bottom topography on the development of trapped filaments has not received a lot of attention, in particular the details of the mechanism is not clear and its sensitivity to different parameters remains to be studied.

[Stern and Chassignet, 2000] showed, using both a $1_{1 / 2}$ and a three-layer isopycnic model, that intrinsic instability was not sufficient to generate detrainment of fluid and eddy-separation from the jet. They concluded that, to generate a blocking wave and detrain water, there was a need for a downstream variation in jet transport, and noted that this variation could happen in the case of alongshore varying topography, but did not investigate further on this point. [Viera and Grimshaw, 1994] studied the evolution of a potential vorticity front over an isolated topography, using a $1_{1 / 2}$ layers quasi-geostrophic model, and showed, that a linearly 
stable jet associated with a potential vorticity front could produce large and pinched off meanders when interacting with bottom topography. [Herbette et al., 2003] have shown that a seamount could interact with a surface intensified eddy and generate filaments (or even split the eddy). Finally, while studying the generation of secondary upwelling fronts along continental slopes [Rossi et al., 2009] found, in one of the experiment with a promontory (see fig. 22 in [Rossi et al., 2009]), that a bottom topography could trap upwelled waters and even observed the formation of a trapped filament extending offshore.

Therefore, we study the evolution of an upwelling front in the presence of an along shore varying topography, in the form of cross-shore coastal promontories. We focus on the formation and trapping of long filaments extending offshore and we base our approach on the potential vorticity analysis used in the papers quoted in the previous paragraph.

The outline is :

- in section 2 (Model and tools) we describe the numerical model and recall some basic principles relating potential vorticity (thereafter $P V$ ) and potential vorticity anomalies (thereafter $P V A)$ to the dynamics;

- in section 3 (Reference experiment) we present a first simulation that illustrates the development of a long filament. In particular we describe how the generation of PVA by the displacement of water columns above the promontory can generate a permanent filament trapped downstream of the promontory;

- in section 4 (Sensitivity study) we study the sensitivity of this mechanism to different regimes and parameters : stable and unstable cases, wind forcing duration, promontory height, width and length, stratification, bottom friction;

- in section 5 (Conclusion) we sum up and discuss our results.

\section{Model and tools}

\subsection{Equations and model}

The model used is an adiabatic version of MICOM (Miami Isopycnic Coordinate Ocean Model) ([Bleck and Boudra, 1986]; [Bleck and Smith, 1990];[Bleck et al., 1992]) modified to include a fourth order scheme in the non-linear advection terms and a biharmonic diffusion operator to improve the PV dynamics ([Winther et al., 2007, Morel and McWilliams, 2001, Herbette et al., 2003]). This model solves the shallow water equations which, for the two-layer configurations considered here, can be expressed as :

$$
\begin{aligned}
\partial_{t} \mathbf{u}_{\mathbf{k}}+\left(\mathbf{u}_{k} \cdot \nabla\right) \mathbf{u}_{k}+f_{0} \mathbf{k} \times \mathbf{u}_{k} & =-\nabla \mathcal{M}_{k}+F_{k}+T_{k}^{w}, \\
\partial_{t} h_{k}+\nabla \cdot\left(\mathbf{u}_{k} h_{k}\right) & =0,
\end{aligned}
$$

where $k$ is the layer number (here, $k=1$ for the top layer and $k=2$ for the bottom one), $\mathbf{u}_{k}=\left(u_{k}, v_{k}\right)$ is the horizontal velocity, $f_{0}=10^{-4} s^{-1}$ is the Coriolis parameter (considered constant here), $h_{k}$ is the thickness of the isopycnal layer $k, T_{k}^{w}$ represents the wind forcing, and $F_{k}$ contains the frictional and viscosity terms (horizontal diffusion is associated with a biharmonic operator with a viscosity that depends on the velocity modulus and deformation tensor, 
see [Winther et al., 2007]). Finally, $\mathcal{M}_{k}$ is the Montgomery potential (pressure along an isopycalface), which can be written :

$$
\mathcal{M}_{k}=\sum_{i=1}^{2} g h_{i}+\sum_{i=1}^{k-1} \frac{\rho_{i}-\rho_{k}}{\rho_{k}} g h_{i},
$$

where $\rho_{i}$ is the density of the isopycnic layer $i$ and $g$ is the gravity acceleration.

\subsection{Configuration and parameters}

The configuration is a periodic zonal channel on an f-plane, with vertical side walls on the northern and southern boundaries. The bottom is flat except near the southern boundaries in the middle of the domain where there exists a promontory. As shown in figure 2, the promontory is composed of a flat plateau of variable height $H_{t}$, length $L_{y}$ and width $L_{x}$, rounded at its offshore edge, and surrounded by a Gaussian slope of a variable extension $d L$ (figure 2). To represent a mid latitude summer thermocline, the surface layer depth at rest $H_{1}=50 \mathrm{~m}$ and the bottom layer depth away from the promontory is $H_{2}^{\infty}=2000 \mathrm{~m}$ for most experiments. The upper layer density is fixed to $\rho_{1}=1000 \mathrm{~kg} / \mathrm{m}^{3}$, the water column stratification is defined by the reduced gravity $g^{\prime}=g\left(\rho_{2}-\rho_{1}\right) / \rho_{1}$. Unless stated otherwise (when testing the sensitivity to the stratification characteristics) $g^{\prime} \simeq 0.01$.

$R_{d}=\sqrt{g^{\prime} H_{1} H_{2} /\left(H_{1}+H_{2}\right)} / f_{0}$ is the Rossby radius of deformation, and $R_{d} \simeq 7 \mathrm{~km}\left(H_{1}=\right.$ $50 \mathrm{~m}, H_{2}^{\infty}=2000 \mathrm{~m}, f_{0}=10^{-4} \mathrm{~s}^{-1}$ and $g^{\prime}=1^{\circ} \%$ o ) for most of the experiments presented below. !!!???YM This value is smaller than the usual Rossby radius of deformation observed in the deep ocean (around $20 \mathrm{~km}$ ). It is however consistent with the upper ocean stratification in summer (when the seasonnal pycnocline has been formed) and corresponds to mid-slope or shelf characteristics. In addition, sensitivity experiments where the Rossby Radius is varied will show that this parameter has a weak influence on the processes studied here (see section 4.2). !!!???YM

The parameters corresponding to the various simulations presented below can be found in table 1 (fixed parameters) and 2 (variable parameters).

\subsection{Potential vorticity and potential vorticity anomaly}

For the shallow water model used here, the potential vorticity for each isopycnic layer is defined as :

$$
P V_{k}=\frac{f_{0}+\zeta_{k}}{h_{k}},
$$

where $\zeta_{k}=\nabla \times \mathbf{u}_{k}=\partial_{x} v_{k}-\partial_{y} u_{k}$ is relative vorticity in layer $k$, and $h_{k}$ is the layer thickness. In the absence of diabatic process, PV is conserved for each fluid particle. PV is also related to the velocity field that can then be calculated by inverting the PV field under the assumption of (cyclo-)geostrophic equilibrium. PV conservation and invertibility are key properties which helped understand and interpret many geophysical fluid processes ([McWilliams and Gent, 1980], [McIntyre and Norton, 1990], [Hoskins et al., 1985], see also [Morel et al., 2006, Rossi et al., 2009] for applications to upwelling dynamics).

$\mathrm{PV}$ is finite at rest and in order to invert it and to calculate the velocity, we use the potential vorticity anomaly $(P V A)$ which is defined in each layer $k$ as the difference between the local $P V$ 


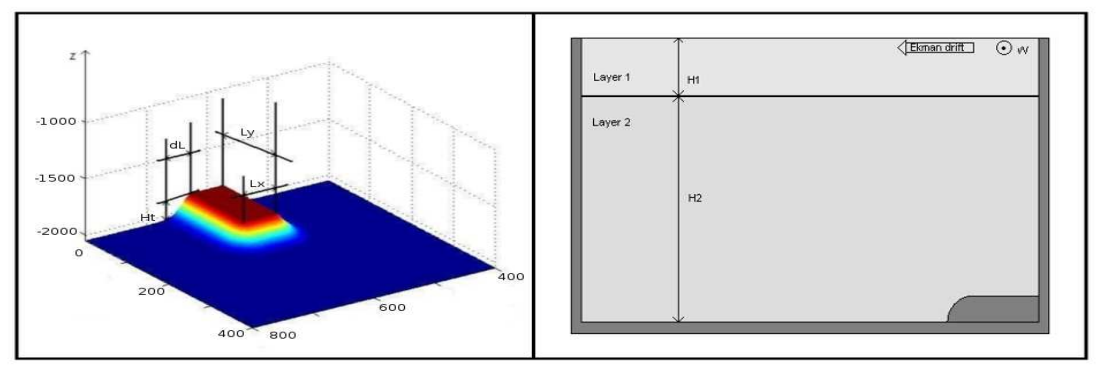

Figure 2: a : Details of the promontory : It is centered on $x=300 \mathrm{~km} . L_{x}$ and $L_{y}$ are respectively the length and width of its flat top, $H t$ its height, and $d L$ is the typical length of its Gaussian sloping sides. $\mathrm{b}$ : Model configuration at rest : a shallow surface layer of depth $H_{1}$ and density $\rho_{1}$ lies over a bottom layer of depth $H_{2}$ and density $\rho_{2}$. The numerical domain is a periodic zonal channel with vertical walls and a flat bottom.

and a reference $P V$ for a state at rest (no current and flat isopycnals) (see [Herbette et al., 2003, Herbette et al., 2005, Rossi et al., 2009]) :

$$
P V A_{k}=H_{k}\left(P V_{k}-P V_{k}^{r e f}\right)=H_{k}\left(\frac{f_{0}+\zeta_{k}}{h_{k}}-\frac{f_{0}}{H_{k}}\right)=\frac{H_{k}}{h_{k}}\left(\zeta_{k}-f_{0} \frac{\delta h_{k}}{H_{k}}\right),
$$

where $H_{k}(x, y)$ is the layer thickness at rest, and $\delta h_{k}=h_{k}-H_{k}$. Notice that we have also multiplied the $P V$ difference by the layer thickness at rest so that $P V A$ is proportional to the vorticity, which makes it easier to analyze. $P V A$ contains the dynamical signal associated with the PV field and the geostrophic velocity field can be inferred from the $P V A$ field. Notice that, as $H_{k}$ is a function of position, contrary to $P V, P V A$ is not conserved for each particle in the presence of a variable bottom topography. It is however directly related to the circulation. The presence of a $P V A$ pole in a layer $k$ is indeed associated with a circulation extending to all layers but intensified in layer $k$ ([Hoskins et al., 1985, Rossi et al., 2009]) : a positive PVA pole being associated with a cyclonic circulation, a negative one with an anticyclonic circulation.

As shown in [Verron and Le Provost, 1985, Herbette et al., 2003], when a current develops above a seamount, two opposite sign eddies appear : an anticyclone trapped above the topographic feature associated with the displacement of low PV water columns from the deep ocean upon the seamount and a cyclone associated with the advection of high PV water columns off the topography. Figure 3) describes this process which is adiabatic and relies on the advection of PV and the formation of PVA poles. It also shows that between the two opposite sign eddies a strong jetlike current is formed.

\subsection{Previous results and general upwelling characteristics}

[Morel et al., 2006] found an exact analytical solution for the geostrophic circulation of a 2-D configuration with a flat bottom and a constant wind forcing $T^{w}$. In practice, $T^{w}=\tau^{w} /\left(\rho_{1} h_{1}\right)$ 

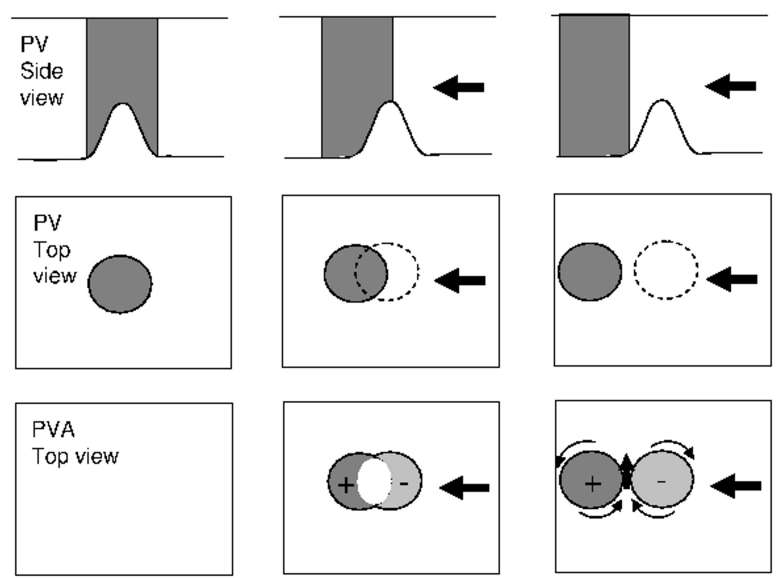

Figure 3: Schematic view of the development of a topographic dipole when a current develops above a seamount. The initial PV structure (top panels for the side view and middle panels for the top view) is associated with high PV above the seamount and lower PV in the deeper ocean; the fluid is initially at rest and the PVA (lower panels) is null. The current exchanges the position of low and high PV water columns which forms positive and negative PVA. An anticyclonic circulation develops above the topography whereas a cyclonic circulation is associated with the high PV water columns detaching from the seamount. The current is intensified between the opposite sign eddies.

(where $\tau^{w}$ is the surface wind stress) is not constant but, with the approximation $T^{w}=\tau^{w} /\left(\rho_{1} H_{1}\right)$, the following formulas provide a good evaluation for the position of the outcropping front and velocity field as a function of the wind stress intensity and the duration of the wind forcing. It is then possible to obtain a stationary basic state current with the desired characteristics by limiting the forcing to a chosen period for a given wind stress intensity.

The distance of the outcropping front from the coast is :

$$
\begin{aligned}
Y(t) & =\min \left\{0,-\frac{T^{w}}{f(1+\delta)}\left(t-t_{0}\right)\right\} \\
t_{0} & =\frac{f R_{d}(1+\delta)}{T^{w}}
\end{aligned}
$$

175

176

if $y \geqslant Y(t)$

where $\delta=H_{1} / H_{2}, t_{0}$ is the time necessary for the lower layer to outcrop at the coast $(y=0) . U_{b}(t)$ 
and $U_{c}(t)$ are the temporal evolution of the barotropic and baroclinic components respectively and can be written as :

$$
\text { if } t<t_{0}
$$

$$
\begin{aligned}
U_{b} & =\frac{\delta T^{w}}{1+\delta} t \\
U_{c} & =\frac{T^{w}}{1+\delta} t
\end{aligned}
$$

$$
\text { if } t \geqslant t_{0}
$$

$$
\begin{aligned}
U_{b} & =\frac{\delta T^{w}}{1+\delta} t \\
U_{c} & =f R_{d} \exp \frac{-Y(t)}{R_{d}}
\end{aligned}
$$

Notice that the maximum current is reached at the outcropping front (for $t \geqslant t_{0}$ ) and is given by:

$$
\begin{aligned}
U_{1}^{\max } & =f R_{d}+\frac{\delta T^{w}}{1+\delta} t \\
& =(1+\delta) f R_{d}+\delta f|Y(t)|
\end{aligned}
$$

Notice that the barotropic mode is spatially constant and only the cross shore spatial structure of the baroclinic mode varies as $\exp \frac{y}{R_{d}}$. In addition, the amplitude of the baroclinic component of the velocity field is limited whereas the barotropic one grows linearly with time (until other processes such as bottom friction become non-neglegible).

The wind stress corresponding to a 30 knots wind $(15 \mathrm{~m} / \mathrm{s})$ is $\tau^{w} \simeq 0.2 N / m^{2}$ and thus we get $T^{w} \simeq 4.10^{-6} \mathrm{~m} / \mathrm{s}^{2}$ (for $H_{1}=50 \mathrm{~m}$ ). Then, the previous formulas show that it takes about $t_{o} \simeq 2$ days for the outcropping front to be generated and after 10 days of wind forcing, the front is located $Y \simeq 35 \mathrm{~km}$ offshore and the maximum velocity at the front is about $U_{1}^{\max } \simeq 70 \mathrm{~cm} / \mathrm{s}$. The barotropic velocity, and the velocity field in the deep layer over most of the domain, are $U_{b} \simeq 8 \mathrm{~cm} / \mathrm{s}$. The characteristics of the upwelling found in the numerical simulations presented below are in very good agreement with these analytical results.

\section{Reference experiment}

The wind forcing was kept constant $T^{w}=\tau^{w} /\left(\rho_{1} H_{1}\right)$ in the reference simulation that we present here.

Figure 4 shows the evolution of the $P V A$ in the upper layer superimposed on the shape of the promontory for the reference experiment (see table 2). During the upwelling development, the upper layer vanishes close to the coast and is replaced by deep waters that reach the sea surface. This area is associated with an infinite PVA in the upper layer (see [Bretherton, 1966]) and is thus delimited by a strong $P V A$ gradient that we use to trace the upwelling front and the development of the upwelling filaments (it is qualitatively comparable to the sea surface temperature front).

The strong $P V A$ gradient associated with the upwelling front becomes evident on the third day of the experiment. It is accompanied by an intense baroclinic surface intensified jet superimposed on a spatially constant barotropic westward flow. The influence of bottom topography 

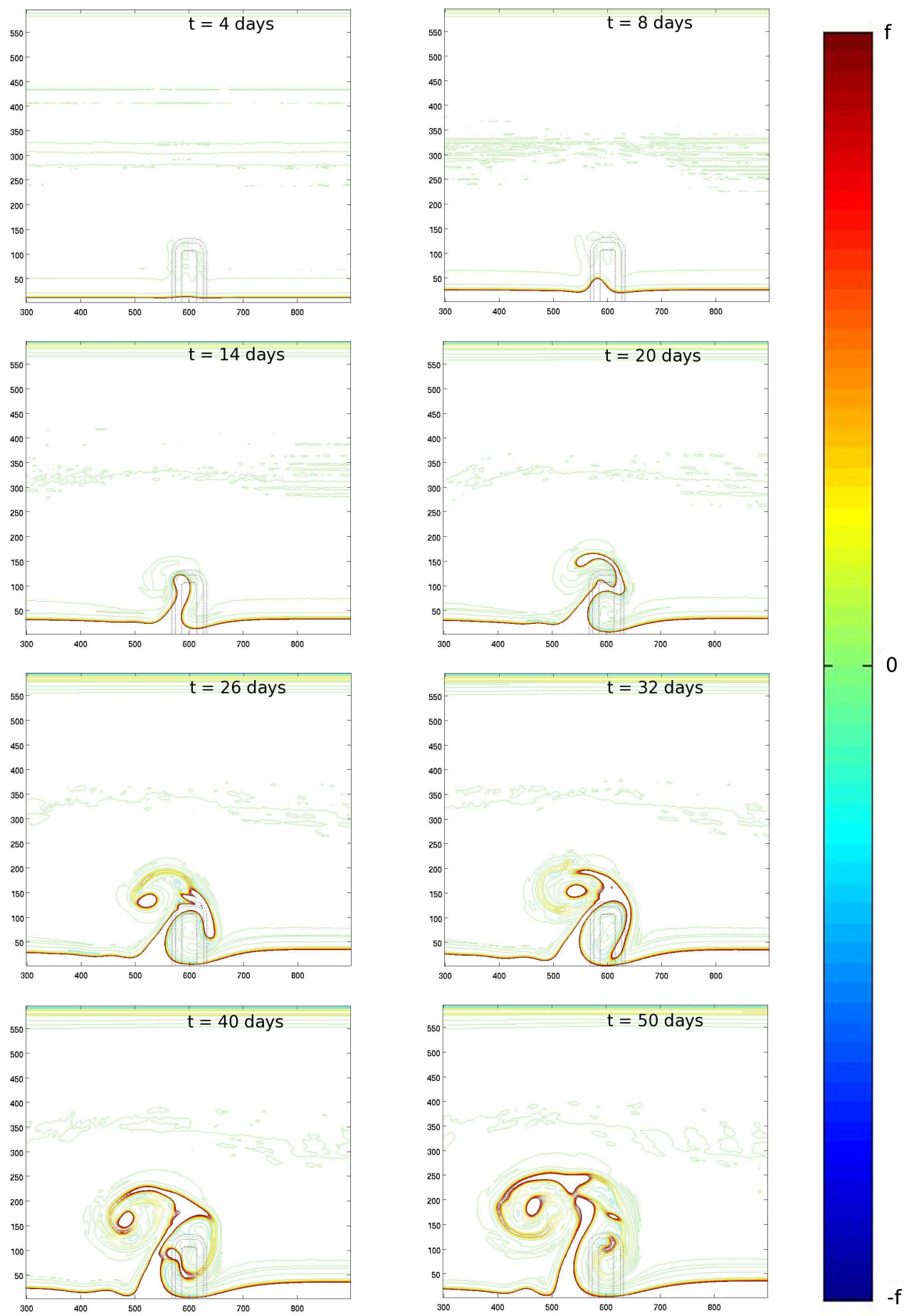

10

Figure 4: Evolution of the PVA in the upper layer for the reference experiment at $t=4,8,14,20,26,32,40,50$ days. The thick red line represents the $P V A=+f$ contour and is a good marker of the upwelling front. The axis are labelled in $\mathrm{km}$. 

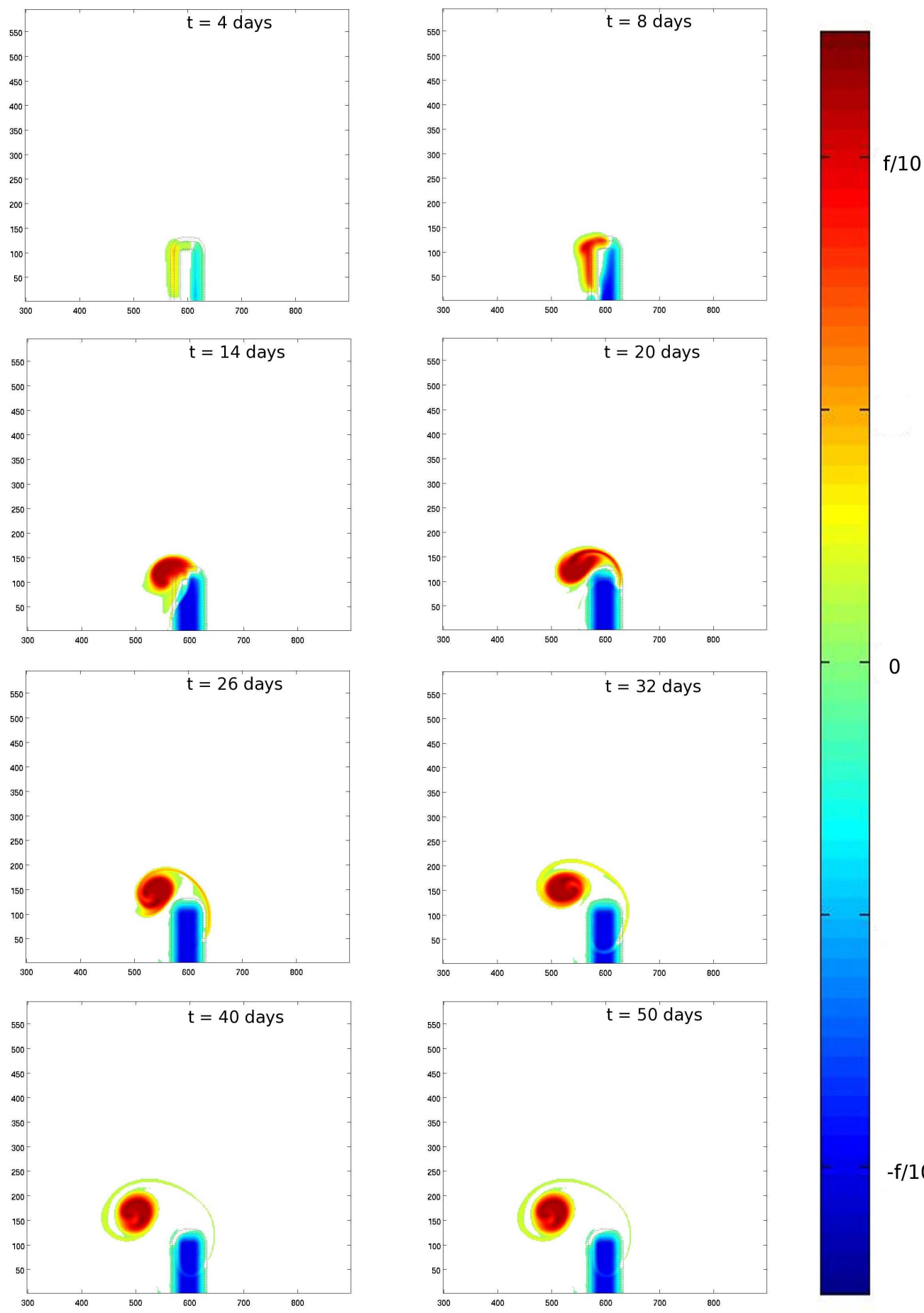

$$
11
$$

Figure 5: Evolution of the PVA in the bottom layer for the reference experiment at $t=4,8,14,20,26,32,40,50$ days. The generation of $P V A$ is visible in the first 10 days. It is then advected from day 10 to day 50 . 

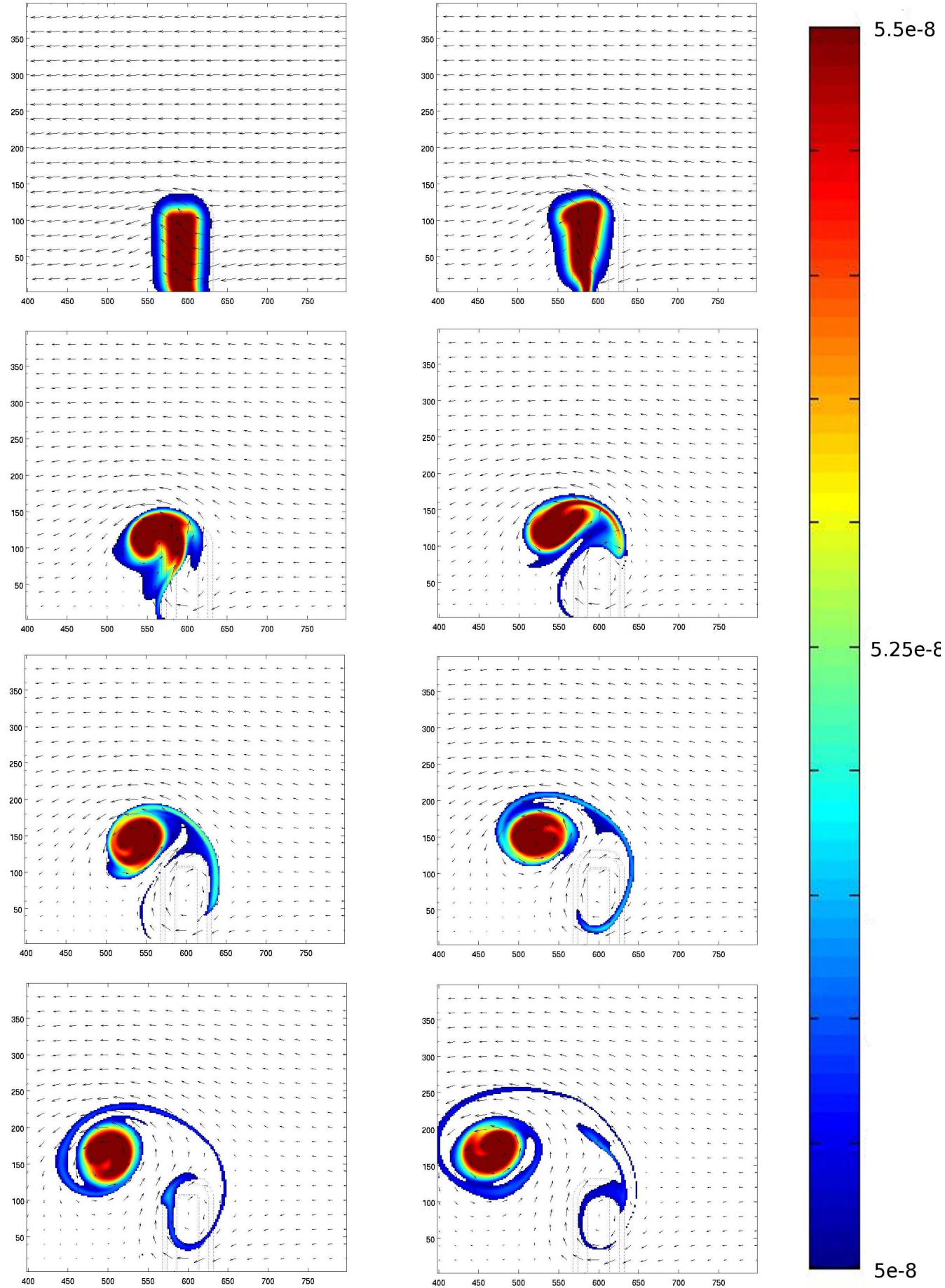

12

Figure 6: Evolution of the bottom layer absolute potential vorticity for the reference experiment superimposed on the velocity field. The high $P V$ pool is visible on the promontory. Note that potential vorticity is strictly conserved and is only advected by the current. Comparison with Fig. 5 also shows that the anticyclonic and cyclonic circulations are associated with the negative and positive PVA poles that develop because of bottom topography, as expected. 
is noticeable after 4 days, when the front begins to form a small meander on the western (downstream) side of the promontory. The initial topographical meander keeps on growing offshore downstream of the promontory. After 12 days of simulation, it starts to roll up anticyclonically around the promontory. At day 16, another branch appears, growing offshore but partly rolling up cyclonically. The meander has then become a thin and elongated filament, surrounded by two counter-rotating recirculations.Its length is about $200 \mathrm{~km}$ and its width about $100 \mathrm{~km}$ near the coast and $20 \mathrm{~km}$ near the tip. It is similar to the 'squirts', observed in all major upwelling systems. After 28 days, the filament is still growing offshore and is about $220 \mathrm{~km}$ long, but its offshore edge has rolled up cyclonically. As shown on Fig. 4, the filament continues to grow until the end of the simulation (it is about $230 \mathrm{~km}$ long after 50 days), corresponding to a mean growth rate of about $5 \mathrm{~km}$ per day for the whole life cycle, consistent with the observed mean value found by [Kostianoy and Zatsepin, 1996]. But the growth rate of the filament is variable during the life cycle, with higher initial growth rates of up to $12 \mathrm{~km} \mathrm{day}^{-1}$ (i.e. $14 \mathrm{~cm} \mathrm{~s}^{-1}$ ) after 10 days, then decreasing until the end of the experiment.

The formation of the filament is very similar to the erosion process of a surface vortex by a seamount studied in [Herbette et al., 2003]. As already explained above, the origin of these counter-rotating eddies can indeed be inferred from the $P V$ and $P V A$ evolution in the lower layer. Indeed, as shown in [Morel et al., 2006, Rossi et al., 2009] a barotropic westward current is generated during the upwelling development. The baroclinic circulation opposes this current in the upper layer, but its extension is of the order of the first internal radius of deformation (about $7 \mathrm{~km}$ here) which is quite small. As a result, water columns move westward over most of the lower layer. As the dynamics is adiabatic here, the initial $P V$ field is simply advected and the positive anomaly associated with the promontory moves downstream (see Fig. 6) replaced by lower $P V$ water columns coming from deeper region. Figure 5 shows that it creates opposite sign $P V A$ (see also [Herbette et al., 2003]): high $P V$ water columns coming from the promontory move in regions with lower $P V$ at rest, forming a positive $P V A$ downstream of the promontory, while low $P V$ water columns move upon the promontory, which is associated with high $P V$ at rest, forming a negative $P V A$ that is being trapped above the promontory. As shown in Fig. 6, this topographic $P V A$ dipole is associated with cyclonic and anticyclonic circulations extending over the whole water column. An offshore jetlike current develops between the two opposite sign PVA poles, which forms the filament.

After 7 days both positive and negative $P V A$ reach a maximum modulus of $\pm 0.1 f$, the negative $P V A$ obviously remains trapped above the topography, maintaining offshore currents on the western side of the promontory which reach about $40 \mathrm{cms}^{-1}$. But the high PVA pole is strongly deformed and propagates offshore and westward under the combined effect of advection and interaction with bottom topography. The topographic $\beta$-drift of a $P V A$ pole along the slope of the promontory scales -in the quasi-geostrophic approximation- as $U_{d r i f t}=\beta_{t} R_{d}^{2}$, with $\beta_{t}=f_{0} \alpha / H_{2}$ where $\alpha=H_{t} / d L$ is the characteristic slope of the promontory, and $R_{d}^{2}$ is the square of the Rossby radius. For the reference experiment, $U_{d r i f t}$ is found to be about $2.5 \mathrm{cms}^{-1}$. The barotropic velocity field associated with a PVA pole in the lower layer can be scaled using the circulation theorem :

$$
\begin{aligned}
U^{-} & \simeq C / 2 \pi l \\
C & =\iint_{13} P V A \frac{h_{2}}{H_{t o t}} d S
\end{aligned}
$$




$$
\simeq \iint_{P} P V A d S
$$

where $C$ is the circulation or total PVA reservoir inside a domain $P, l$ is the distance from the center of the PVA pole, $H_{t o t}=H_{1}+H_{2}$ and $d S$ is the surface element. If we assume that all water columns above the promontory have been replaced by water columns coming from the deeper ocean, the negative PVA forming above the promontory is given by :

$$
P V A=-\frac{f H_{p}(x, y)}{H_{2}^{\infty}}
$$

where $H_{p}(x, y)$ is the the promontory height.

The total circulation associated with the negative $P V A$ of the promontory, over the domain $P$ is then

$$
\begin{aligned}
C & =-\frac{f}{H_{2}} \iint_{P} H_{p}(x, y) d S \\
& \simeq-\frac{f}{H_{2}} H_{t}\left[L_{x} L_{y}+d L\left(L_{x}+L_{y} / 2\right)\right]
\end{aligned}
$$

For the positive pole, the calculations are similar : the PVA reservoir, and thus circulation, is exactly the opposite of the negative one above the promontory (water columns are exchanged between the deep ocean and the promontory). As the effect of both PVA poles superimposes, the maximum barotropic current between both poles is thus roughly given by :

$$
\begin{aligned}
U_{\max }^{\text {jet }} & \simeq 2 C / 2 \pi l \\
C & \simeq \frac{f}{H_{2}} H_{t}\left[L_{x} L_{y}+d L\left(L_{x}+L_{y} / 2\right)\right]
\end{aligned}
$$

where $l$ is the mid distance between both pole centers.

When both PVA poles are well developed, $l \simeq 30 \mathrm{~km}$ and $U_{\max }^{\text {jet }} \simeq 36 \mathrm{~cm} / \mathrm{s}$, which is the order of magnitude of the maximum offshore current observed downstream of the promontory $(40 \mathrm{~cm} / \mathrm{s})$. These modelled velocities are in very good agreement with what have been observed in-situ in the IPUS area and also in other upwelling regions (see [Sanchez et al., 2008]).

Notice that the estimation of $U_{\max }^{\text {jet }}$ or $U^{-}$is only correct in the case of circular $P V A$ structures, or far enough from the structure so that shape effects become negligible. Here, this is obviously not verified, but, using this simple scaling can give us a good insight of the order of magnitude of the velocity associated with the topographic PVA pole development and its sensitivity to the promontory characteristics.

In summary, an anticyclonic circulation is generated and trapped above the promontory by advection of low $P V$ over the topography, forming a negative $P V A$ pole. A cyclonic circulation also forms because of advection of high $P V$ from the promontory into a deeper environment. This forms a trapped topographic dipole associated with a strong offshore current that generates the filament and its well known 'squirt' or 'mushroom' shapes (see [Strub et al., 1991]). The strength of the current depends on the total PVA reservoir of the promontory.

Finally, notice that, even though the initial topographic cyclone slowly separates from the trapped anticyclone, because of the outcropping and vanishing of the upper layer, the meander and filament are themselves also associated with an equivalent high PVA (see [Bretherton, 1966]) reinforcing and maintaining a cyclonic circulation on the downstream side of the negative $P V A$ pole. 


\section{Sensitivity experiments}

To strengthen the physical relevance of the mechanism described above and to assess the respective importance of the various parameters and characteristics of the configuration, a set of sensitivity tests was performed. Here we focused on the stability of the front, the forcing duration time, of the promontory characteristics (width, length, height and slopes), of the stratification and of bottom friction. For comparison of the various model output, we take as a reference time the $t=42$ days (6 weeks) output, and use the PVA maps as a qualitative indicator of the efficiency of the model configuration to produce long, coherent and trapped filaments.

\subsection{The stability of the front}

The reference run showed that the sole presence of the topography allowed the development of a long filamentary structure reaching as far as $230 \mathrm{~km}$ offshore. However, mixed barotropic-baroclinic instability is a well known feature of upwelling currents [Shi and Roed, 1999] and has sometimes also been referred to as the main process for filament formation [Haidvogel et al., 1991]. It is thus important to evaluate the relative influence of topography and intrisic instability on the development of the long filament.

Baroclinic instability can only develop when there exists opposite sign potential vorticity gradients or PVA (see [Charney and Stern, 1962]). The outcropping front is associated with positive $P V A$. As shown by [Morel et al., 2006], negative $P V A$ is generated along the upwelling front (see Fig. 7 below) because as isopycnic surfaces bend upward they enter the region influenced by the wind stress. A wind stress curl then exists along isopycnic levels which has been shown to necessarily form negative $P V A$ (the formation of negative $P V A$ by the wind has also been studied in [Thomas, 2005]). In the simple 2-layer configuration used here, this effect is associated with the fact that $T^{w}=\tau^{w} /\left(\rho_{1} h_{1}\right)$ varies with the layer depth $h_{1}$.

In the reference experiment, the possibility of the flow to become baroclinically unstable has thus been suppressed by modifying the distribution of the wind forcing : $T^{w}=\tau^{w} /\left(\rho_{1} H_{1}\right)$ provides a constant wind stress so that the upwelling still develops but the dynamics remains adiabatic and the PV field is conserved. As a result, no negative PVA is formed along the front and no baroclinic instability can develop.

In the present test, we use the actual wind forcing $T^{w}=\tau^{w} /\left(\rho_{1} h_{1}\right)$. Figures 7 and 8 show the evolution of the $P V A$ in the upper layer and in the lower layer respectively. In comparison with the reference experiment (see Fig. 4 and 5), negative PVA is develops along the upwelling front. This negative PVA strip interacts with the positive PVA associated with the outcropping forming new small-scale meanders, with wavelengths of $30 \mathrm{~km}$ after 10 days (notice the association of the small upwelling front meanders with small negative PVA poles). These small-scale meanders are associated with baroclinic (or sometimes called frontal) instabilities ([Barth, 1989 a, Barth, 1989 b, Morel et al., 2006, Capet and Carton, 2004, Killworth, 1980]) but are neither trapped nor forming long filaments. They indeed propagate along the upwelling front, re-enter the domain on the eastern side and only very slowly develop after their initial growing. After 5 weeks of experiment, their offshore extension is less than $50 \mathrm{~km}$ (from the front).

The impact on the main filament is also minor: the positive and negative $P V A$ poles still develop in the bottom layer and their time evolution is not significantly modified. The surface filament is very similar to the one observed in the stable case and it extends as far offshore. The only noticeable difference, apart from the absence of the small amplitude meanders along the front, is that the topographic filament is here truncated by the small scale eddies and also appears 

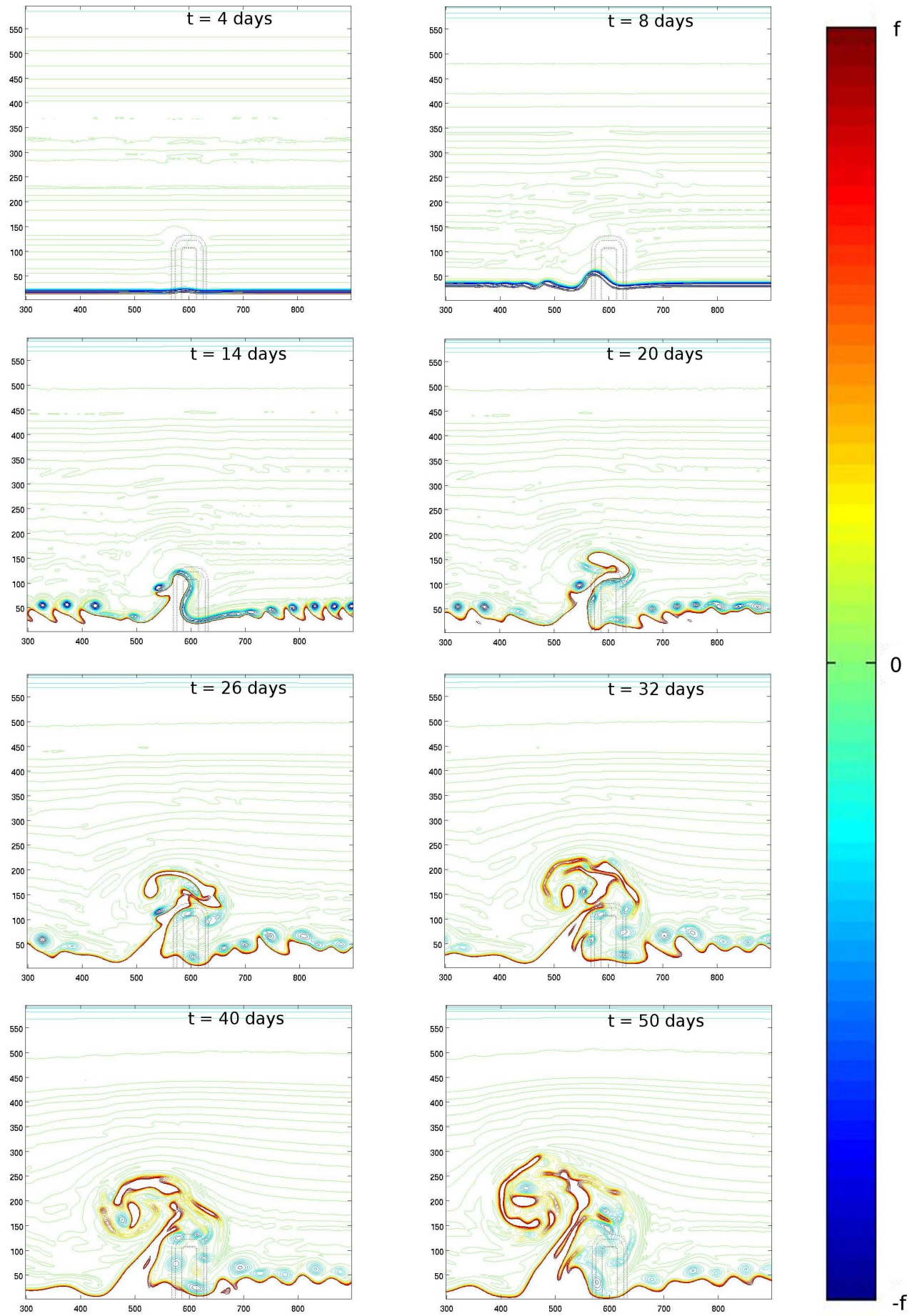

16

Figure 7: Evolution of the $P V A$ in the upper layer for the unstable experiment at $t=4,8,14,20,26,32,40,50$ days Notice the additional smaller meanders, but the formation of the large filament is the same as in Fig. 4. 

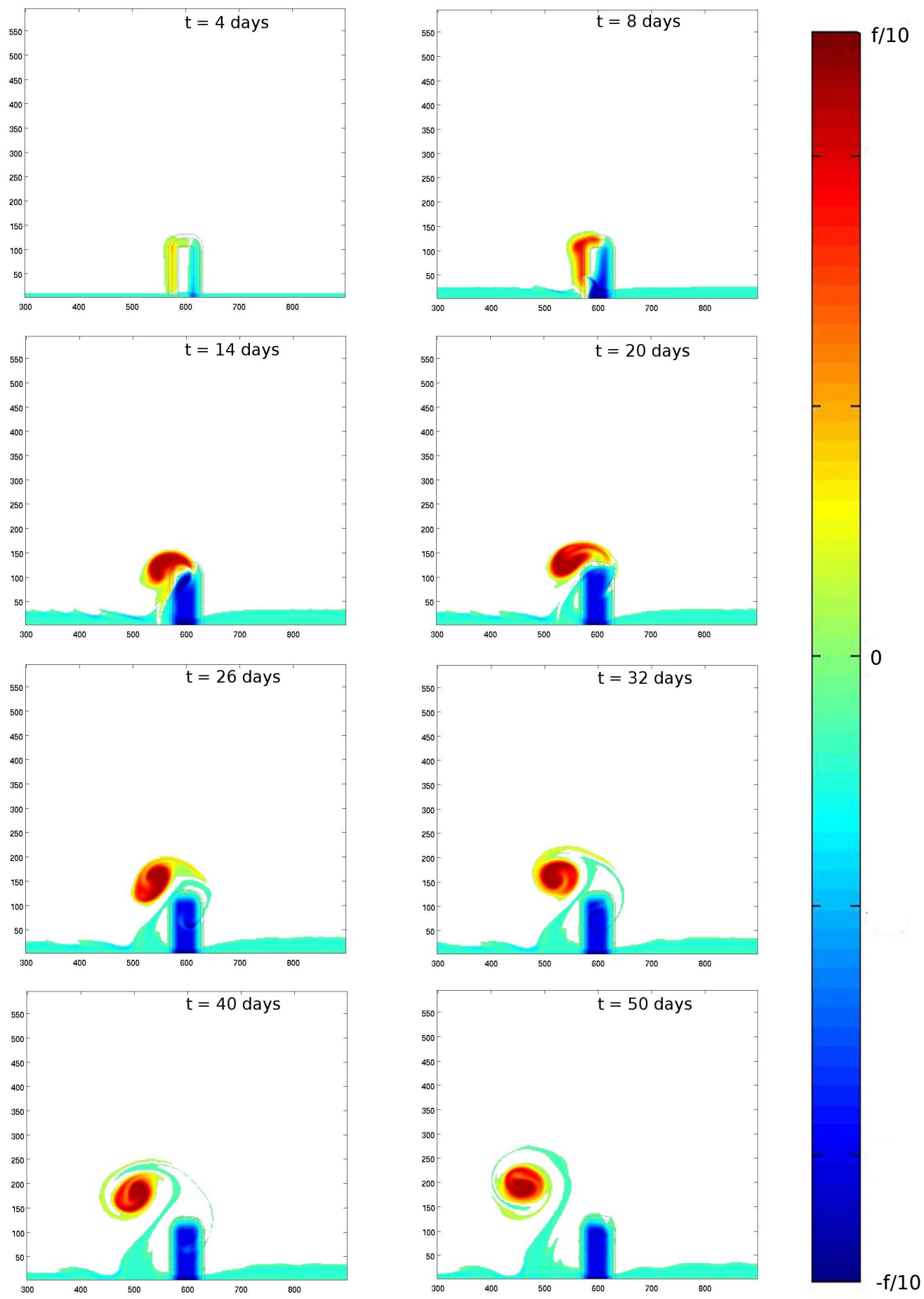

17

Figure 8: Evolution of the PVA in the bottom layer for the unstable experiment at $t=4,8,14,20,26,32,40,50$ days. 
slightly larger at its base. This proves that, at least in our simplified configuration, intrinsic baroclinic instability has little influence on the formation of the long filament and that the main mechanism is associated with topography, as described above.

The stable front configuration is also of particular interest because the dynamics is adiabatic and $P V$ is conserved following fluid parcels in all layers and can be used as a tracer. For this reason, and because we have shown that no substantial difference existed in the formation of the filament, we keep the stable front configuration as our reference experiment for the following sensitivity tests that will thus be carried with the modified and constant wind forcing.

\subsection{The influence of the stratification characteristics}

In addition to the reference experiment $\left(g^{\prime}=0.01, H_{1}=50 \mathrm{~m}\right)$, six additional experiments were performed to evaluate the influence of the stratification characteristics on the dynamics of the topographic filament : 3 experiments varying $g^{\prime}\left(0.005,0.02\right.$ and $\left.0.03 \mathrm{~ms}^{-2}\right)$, and 3 experiments varying $H_{1}(25,100$, and $200 \mathrm{~m})$. It may seem redundant to vary both parameters (as they both influence the Rossby radius) but we finally found out that their respective influence on the upwelling front evolution is quite different.

Figure 9 represent the upper layer PVA after 42 days for different values of $g^{\prime}$ and shows only modest modification of the filament. This is not entirely surprising as the density jump mostly influences the baroclinic currents in the vicinity of the front via the Rosbby radius of deformation. Topographic eddies are formed and influence the dynamics through the barotropic circulation, which is not modified. In addition, the position of the upwelling front is also only slightly affected by a modification of $g^{\prime}:$ the offshore displacement is not modified and only the initial time at which the outcropping front forms depends on this parameter.

Varying $H_{1}$ (Fig. 10) does not modify the lower layer dynamics either (see the similarities of the PVA structure in the lower layer after 42 days on the right panels of Fig. 10). However, since it also plays a role in the strength of the wind forcing $\left(T^{w}=\tau^{w} /\left(\rho_{1} H_{1}\right)\right)$, it strongly modifies the position of the upwelling front, which forms later and extends more slowly for deeper thermoclines (larger $H_{1}$ ). The differences in the filament evolution with different $H_{1}$ is thus the result of the time lag between the upwelling front evolution associated with $H_{1}$ and the distribution of the topographic eddies when the outcropping first forms. As a result, the advective effect of the topographic eddies on the upwelling front is in general simply delayed. The time period necessary for the upwelling front to be formed is $t_{o} \simeq 0.7,2,6$ and 16 days for $H_{1}=25,50$, 100 and $200 \mathrm{~m}$ respectively. As a result, for the duration of the wind forcing considered here (10 days), varying $H_{1}$ does not strongly modify the filament except for the deepest thermocline (here associated with the case $H_{1}=200 \mathrm{~m}$ ) for which the upwelling front is not formed and no filament is then visible (see Fig. 10 lower panel). Interestingly, the final offshore extent has close values for the all other experiments.

Finally notice that the experiment where $g^{\prime}$ is varied and the experiment where $H_{1}$ is varied have different Rossby radius of deformation : $R_{d}=5,7,10,14 \mathrm{~km}$, for $H_{1}=25,50,100,200$ $\mathrm{m}$ respectively (or $g^{\prime}=0.005,0.01,0.02$ and 0.03 respectively). This underlines again that the important mechanisms for the filament development is the barotropic circulation and the formation of the upwelling front. In our configuration the development of the filament is mainly controlled by the bottom layer $P V A$ evolution which is almost insensitive to $R_{d}$.

\subsection{The forcing duration time}

We here study the effect of a variation of the wind forcing duration time. As seen above, the wind forcing acts both on the offshore front position and the velocity strength, especially the one 

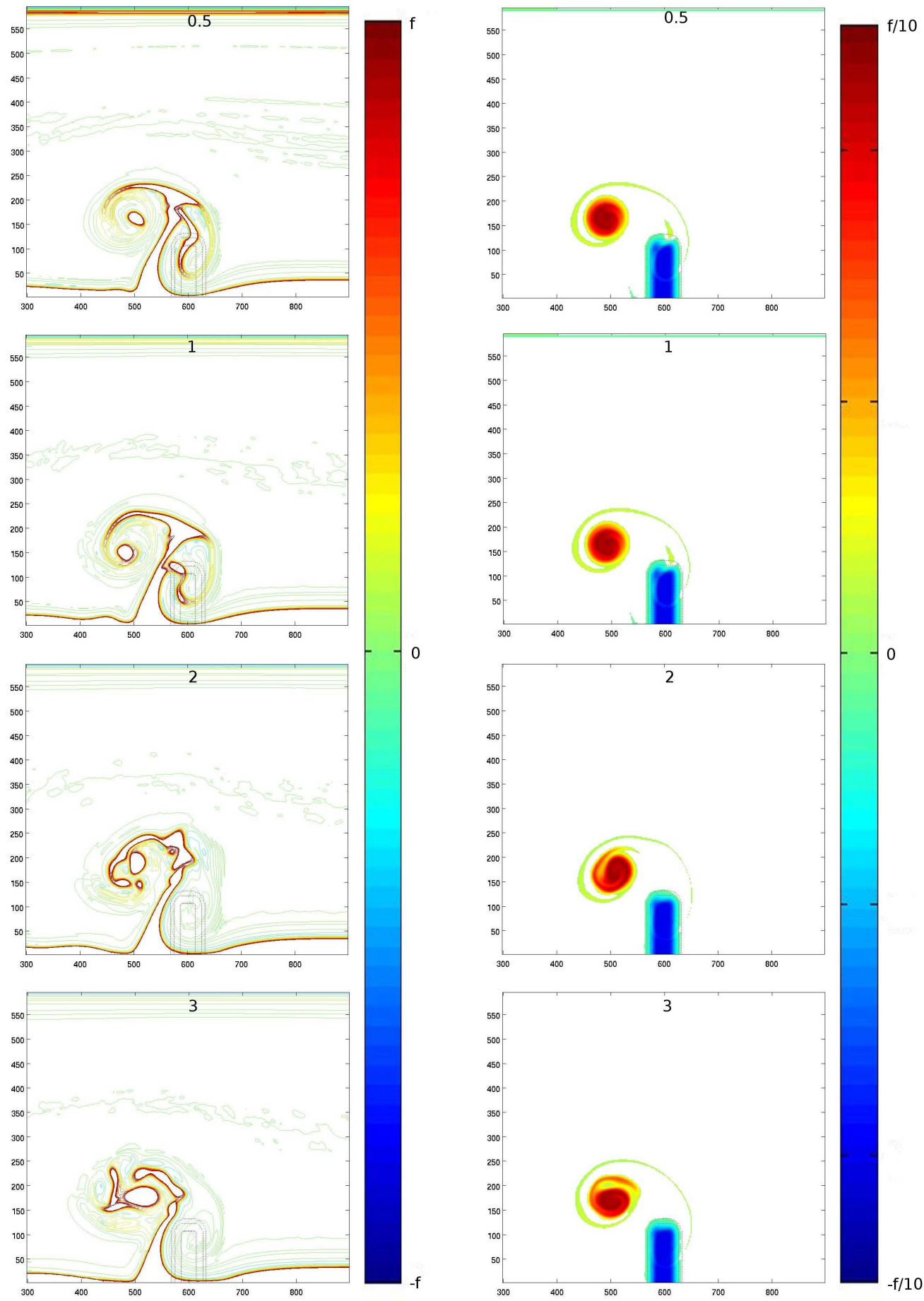

19

Figure 9: Maps of PVA in the upper (left hand panel) and bottom (right hand panel) layers at $t=42$ days for the !!! $g^{\prime}=0.005,0.01,0.02$ and $0.03 \mathrm{~ms}^{-2}$ experiments. !!! 

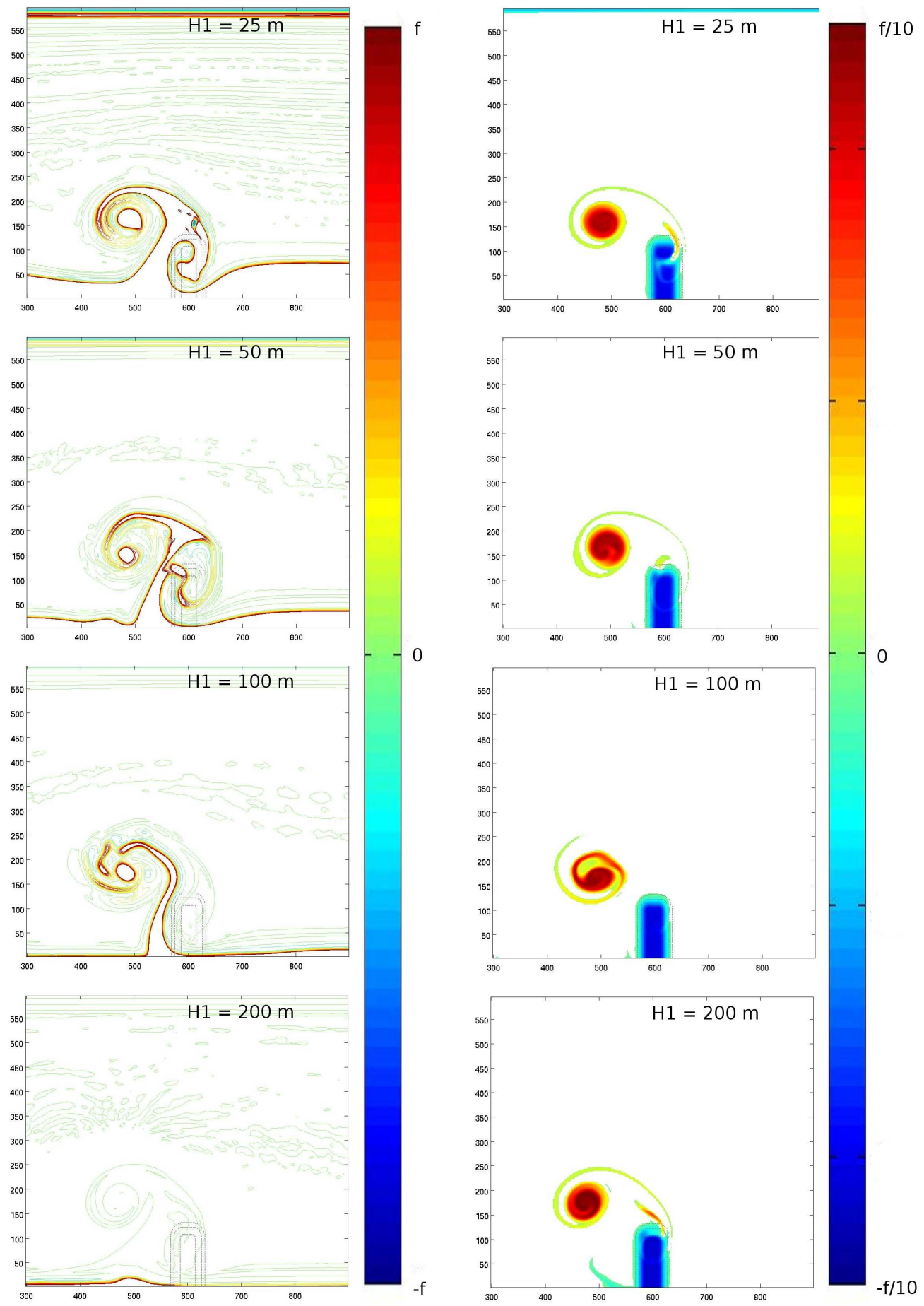

20

Figure 10: Maps of $P V A$ in the upper (left hand panel) and bottom (right hand panel) layers at $t=42$ days for the $H_{1}=25,50,100,200 m$ experiments. 


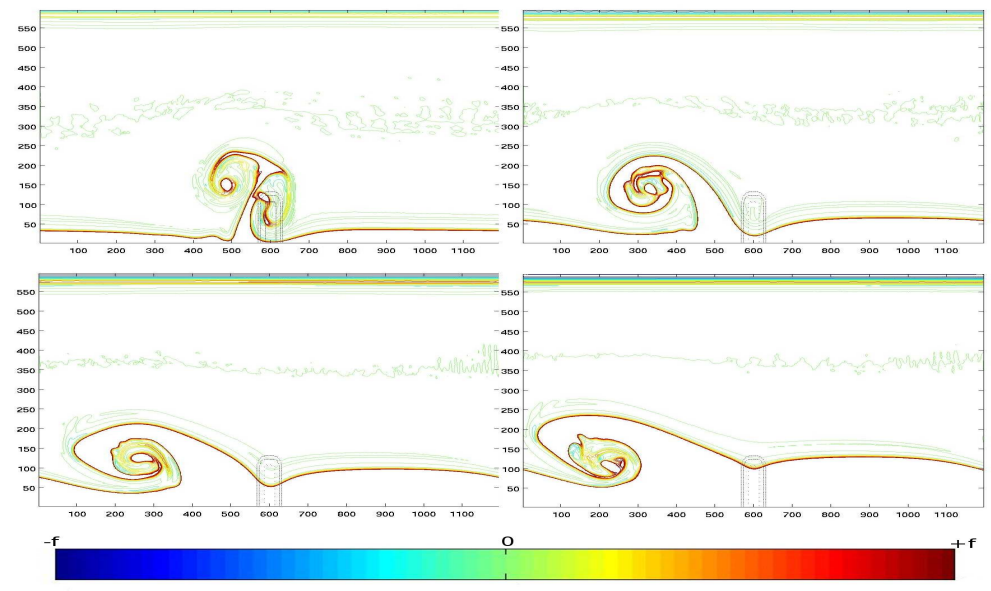

Figure 11: Maps of PVA in the upper layer at $t=42$ days for the 10,20,30 and 40 days of wind forcing cases.

of the barotropic current.

Figure 11 shows the $P V A$ front after 42 days of experiments in the upper layer for forcing durations of 10 (reference experiment), 20,30, and 40 days. There still exists a trapped filament that extends far offshore downstream of the promontory, but its characteristics drastically depend on the forcing duration : it becomes thicker and bends downstream when the wind blows for a long time.

In fact, increasing the wind forcing duration does not substantially modify the formation of the PVA in the bottom layer but induces stronger barotropic current directed downstream. This intensified upwelling can mask the topographic circulation and the offshore jet. In addition, as the barotropic current increases, the positive pole becomes quickly advected downstream and only shortly interacts with the negative $P V A$ pole on the topography. As a result, the upper layer $P V A$ front rolls up cyclonically around the bottom layer positive $P V A$ pole and is entrained downstream, giving it a breaking wave like shape. When increasing the forcing duration time, the distance from the front and jet to the coastal wall increases, while the offshore distortion of the front is less obvious, since its initial position almost reaches the offshore edge of the promontory (see the 40 day forcing case).

Notice that according to Eq. 13 and 15 the maximum barotropic velocity is roughly given by $U_{b}^{\max } \simeq 8,6.10^{-3} t_{d}$, where $t_{d}$ is the duration time of the wind forcing in days. We thus get $U_{b}^{\max } \simeq 8,6 \mathrm{~cm} / \mathrm{s}$ for 10 days and $U_{b}^{\max } \simeq 34,4 \mathrm{~cm} / \mathrm{s}$ for 40 days, which is stronger than the offshore advection associated with the topographic eddies. Notice such barotropic currents are far beyond what is observed, at least offshore the continental shelf, and that in practice, bottom friction keeps the barotropic velocity from reaching such values. 


\subsection{The promontory height}

As the main process proposed here for the development of an upwelling filament is the generation of topographic $P V A$ in the bottom layer associated with the existence of a promontory, it is important to detail how the shape and size of the latter can affect $P V A$ generation and thus filamentation. The maximum $P V A$ and the strength of the topographic eddies are proportional to the height of the promontory which is thus a key parameter.

Six experiments were carried out with different promontory heights : $H_{t}=50,100,300,500$, 1000 and $1500 \mathrm{~m}$, to be compared with the $200 \mathrm{~m}$ of the reference experiment. Figure 12 shows the upper and lower layer PVA field after 42 days for the 50, 200, 500, and $1500 \mathrm{~m}$ experiments. For small topographies $(H t=50 \mathrm{~m})$, the filament forming in the upper layer along the front has a much smaller offshore extension, is less pinched off and its tip is advected downstream. In fact, the effect is the same as for the influence of the forcing duration discussed above: the topographic circulation becomes much smaller than the upwelling current (the offshore current is about $10 \mathrm{~cm} \mathrm{~s}^{-1}$ for $\mathrm{Ht}=50 \mathrm{~m}$, to be compared with the reference experiment where it is about $40 \mathrm{~cm} \mathrm{~s}^{-1}$ ). The positive $P V$ pole is quickly advected downstream and the offshore current is masked by the upwelling current giving the filament a breaking wave shape and limiting its offshore extension.

The $H_{t}=500$ and $H_{t}=1500 \mathrm{~m}$ experiments (see Fig. 12 left panels) show that after 42 days of experiment, the filament is also much reduced in comparison with the reference experiment $\left(100 \mathrm{~km}\right.$ for $H_{t}=1500 \mathrm{~m}$, and $140 \mathrm{~km}$ for $\left.H_{t}=500 \mathrm{~m}\right)$. The limiting factor for large $H_{t}$ is associated with the difficulty for water columns to climb on or leave the topography. Indeed, most of the positive and negative $P V A$ in the bottom layer remains trapped on the slope (see Fig. 12 right panels). As a result, instead of forming two strong opposite sign $P V A$ poles that locally reinforce the offshore circulation, $P V A$ of both signs mix on the promontory evolving into a complex pattern of multiple small poles with few coherence. The overall integrated $P V A$ and circulation associated with the topographic eddies is then much reduced. In fact, as already found by [Herbette et al., 2003] in the case of a vortex interaction with a seamount, the topographic circulation can not be much stronger than the background velocity and the PVA creation is limited. Figure 13 shows the maximum extent of the filament for the 7 experiments. Similarly to [Herbette et al., 2003]'s optimum value of seamount height for vortex erosion, there exists an optimal promontory height for the filament extension which corresponds here to the reference experiment : $H_{t}=200 \mathrm{~m}$.

\subsection{The promontory width}

Three experiments were carried out to test the sensitivity of the filament formation to the width of the promontory (parameter $L_{x}$ ). We tested $L_{x}=0 \mathrm{~km}$ (Gaussian ridge), $20 \mathrm{~km}$ (reference experiment), 50, and $100 \mathrm{~km}$ (see Fig. 14). This parameter mostly affects the PVA reservoir and strength of the circulation associated with the topographic eddies. The evolution of $P V A$ in the bottom layer for small $L_{x}$ exhibits similarities with the reference experiment, with a generation of negative $P V A$ on the upstream part of the promontory in the first week of experiment, fully invading it after 10 days, and a generation of positive $P V A$ downstream of the ridge, quickly evolving into a cyclonic vortex detaching from the offshore edge of the ridge. In fact, in the case of small $L_{x}$, most of the $P V A$ reservoir is contained along the promontory slope, not above the plateau, and the results are then obviously not sensitive to this parameter in this case.

Increasing $L_{x}$ increases the PVA reservoir and the potential strength of the topographic eddies. The $L_{x}=50 \mathrm{~km}$ and $100 \mathrm{~km}$ cases show a strong rolling up of the positive PVA and of the filament 

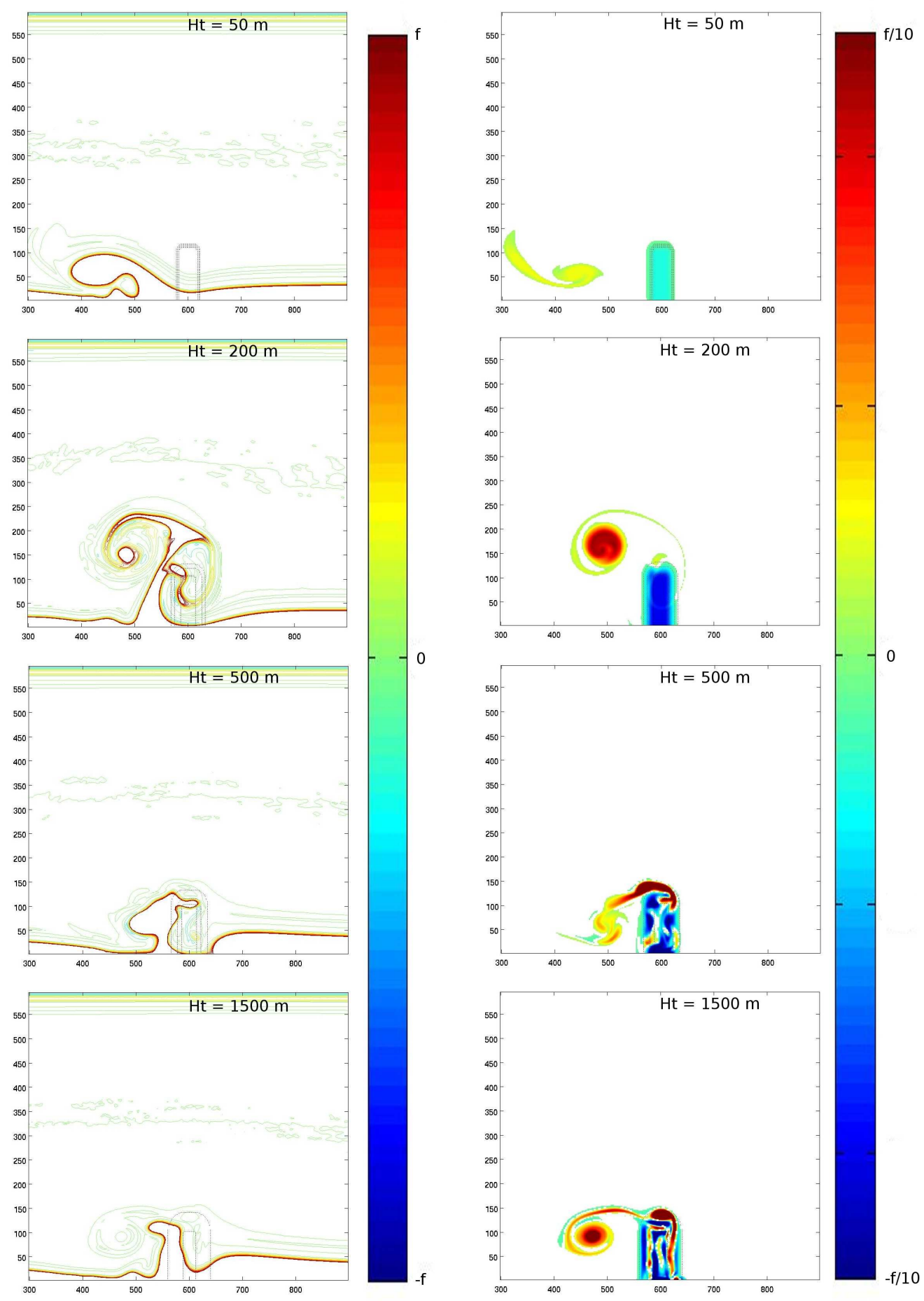

23

Figure 12: Maps of $P V A$ in the upper (left hand panel) and bottom (right hand panel) layers at $t=42$ days for the $H_{t}=50,200,500$ and $1500 m$ experiments. 


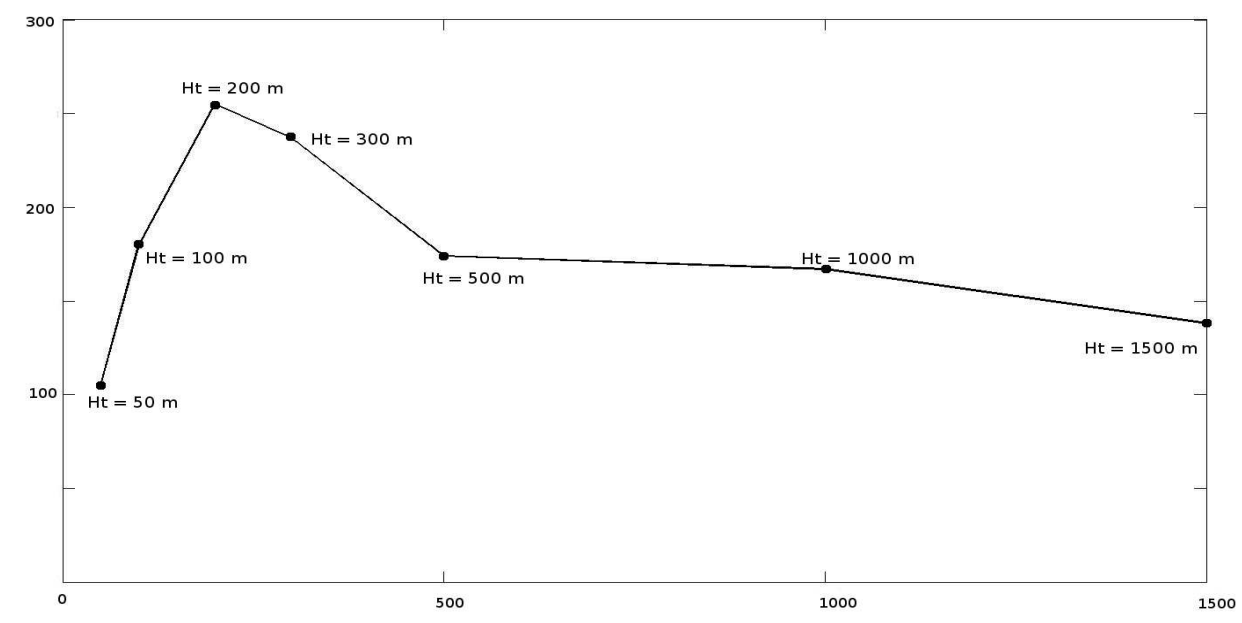

Figure 13: Offshore extent of the filament after 50 days of experiment for the $H t=50,100,200,300,500,1000$ and $1500 \mathrm{~m}$ cases. The $\mathrm{x}$ axis is $H_{t}(\mathrm{kms})$ and the y axis is offshore distance $(\mathrm{kms})$.

around the promontory. The PVA structure also exhibits a multipolar structure and the filament has multiple branches that do not extend very far offshore. In fact, strong topographic eddies leads to complex non linear interactions between the opposite sign PVA poles. The position and shape of the negative PVA is fixed and remains trapped above the promontory, whereas the positive one is advected and deformed by the total velocity field that develops in the lower layer. The latter effect is a combination between the barotropic circulation associated with the upwelling development, which is spatially constant, and the anticyclonic eddy, which varies spatially and can induce strong deformations. When the PVA reservoir increases, the effect of the negative PVA pole dominates the positive PVA pole and filament dynamics which are advected anticyclonically around the promontory and deformed. This greatly reduces the total length of the filament.

As a result, the width of the promontory also plays an important role in the development of a coherent filament structure in the upper layer and again, there exists an optimum value for the width of the promontory. This is shown in Fig. 15 where the offshore extension of the topographic filament is plotted for various choices of $L_{x}$. The optimum value is $L_{x}=20 \mathrm{~km}$ (reference experiment) for the present configuration.

\subsection{The side slopes}

In order to evaluate the importance of topographic $\beta$-effect in the offshore displacement of the positive $P V A$, three experiments were performed with different margin lengths for the promontory : $d L=0,10,20$ (reference experiment) and $40 \mathrm{~km}$. This parameter acts on the PVA reservoir (with close similarities with $L_{x}$ ) but also on the topographic slope and $\beta$-effect. The previous choices for $d L$ corresponds to slopes $\tan \alpha=\infty, 2.10^{-2}, 10^{-2}, 5.10^{-3}$ respectively.

Figure 16 shows upper and lower layer $P V A$ maps at $t=42$ days for the different margin lengths. As could be expected, the influence of $d L$ is similar to $L_{x}$ : above a critical value, 

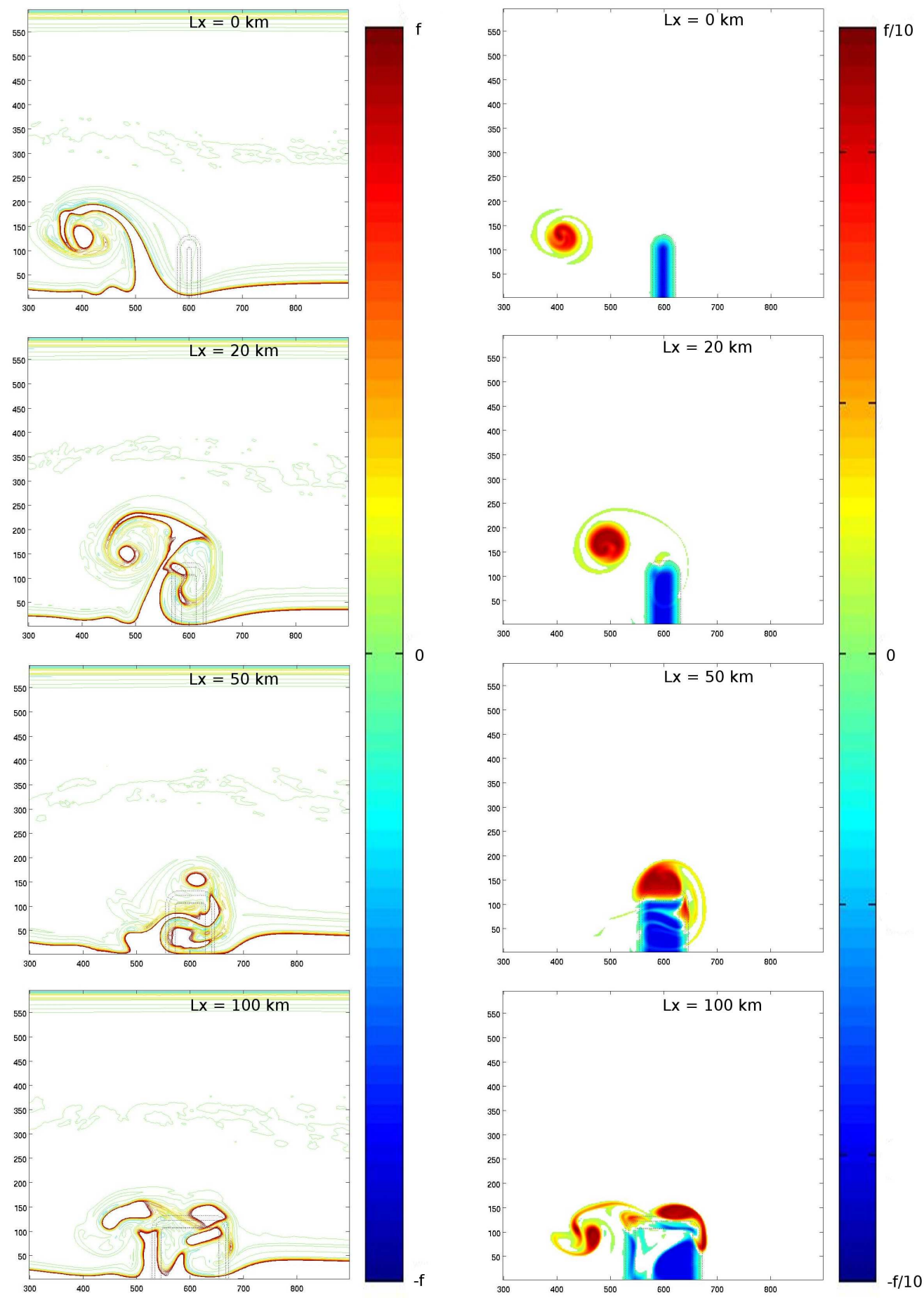


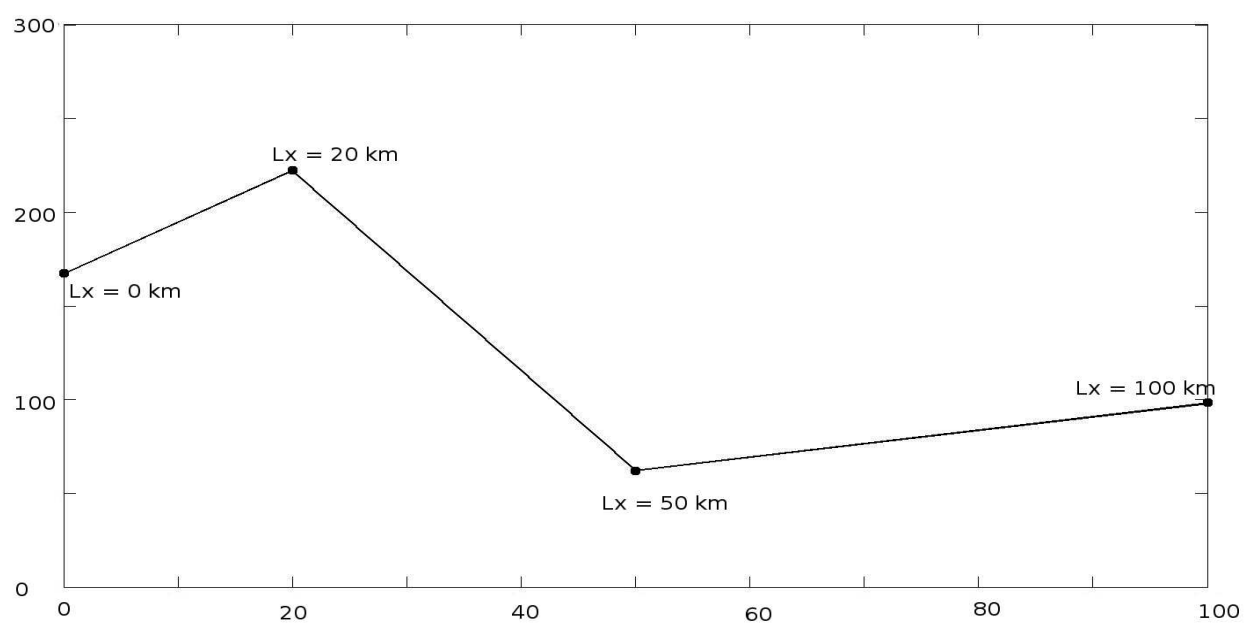

Figure 15: Offshore extent of the filament after 50 days of experiment for the $L_{x}=0,20,50$ and $100 \mathrm{~km}$ cases. The $\mathrm{x}$ axis is $L_{x}(\mathrm{kms})$ and the y axis is offshore distance $(\mathrm{kms})$.

the total circulation is dominated by the negative PVA pole and the filament wraps around the promontory. Despite very different values for the topographic slope and $\beta$-effect, the similarities between Fig. 16 and 14 are striking.

This confirms that the topographic $\beta$-effect has a minor impact on the dynamics, and that the important parameter in the generation of coherent and trapped filaments is the total amount of PVA over the promontory.

\subsection{The promontory length}

As discussed above, anisotropy in the shape of the promontory can also modify the structure and strength of the topographic circulation. The sensitivity of the results to the promontory length $L_{y}$ was thus studied with $L_{y}=50 \mathrm{~km}, L_{y}=100 \mathrm{~km}$ (reference experiment), $L_{y}=150 \mathrm{~km}$ and $L_{y}$ $=200 \mathrm{~km}$.

Figure 17 shows the structure of the $P V A$ after 42 days in both layers and for the different $L_{y}$. The offshore extension of the bottom layer negative $P V A$ pole obviously follows $L_{y}$ and also drives the length of the filament which always extends further than the promontory. The $L y=200 \mathrm{~km}$ case shows that there exists a maximum length of the filament over which it breaks, so that very long promontories are not necessarily the most efficient ones. This is underlined in Fig. 18 which shows the maximum offshore extension of the filament as a function of $L_{y}$. The optimal value is here around $150 \mathrm{~km}$. Another particular feature for long promontories $\left(L_{y}=200\right.$ $\mathrm{km}$ ), is that the filament no longer rolls up around the positive $P V A$ pole as it drifts far offshore, its base is much wider and its offshore shape much thinner.

\subsection{Bottom friction}

Two experiments were performed adding a bottom friction term to the reference configuration. Figure 19 shows the $P V A$ in the upper layer (left hand panel) and the $P V$ in the bottom layer at 

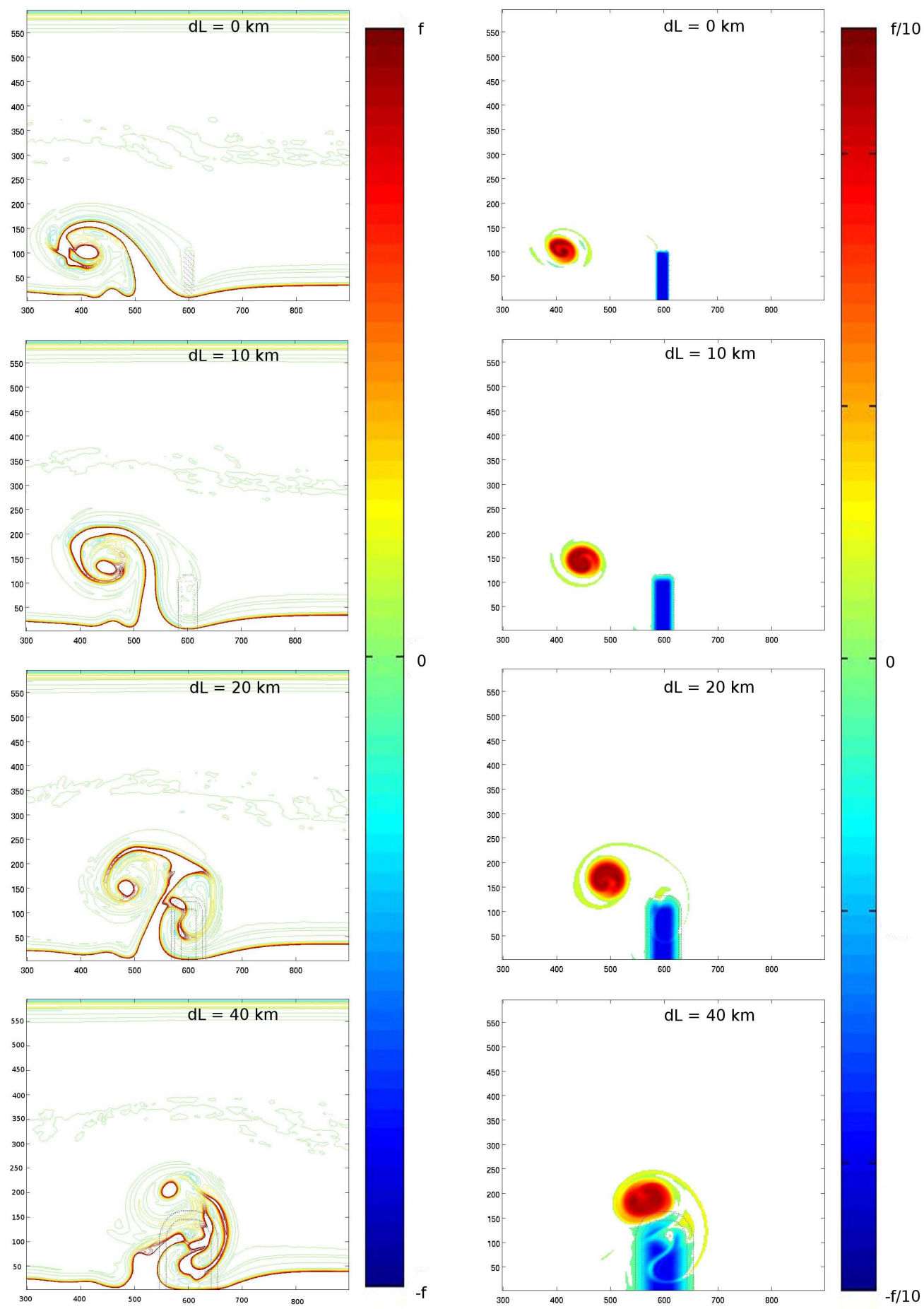

27

Figure 16: Maps of $P V A$ in the upper (left hand panel) and bottom (right hand panel) layers at $t=42$ days for the $d L=0,20,50$ and $100 \mathrm{~km}$ experiments. 

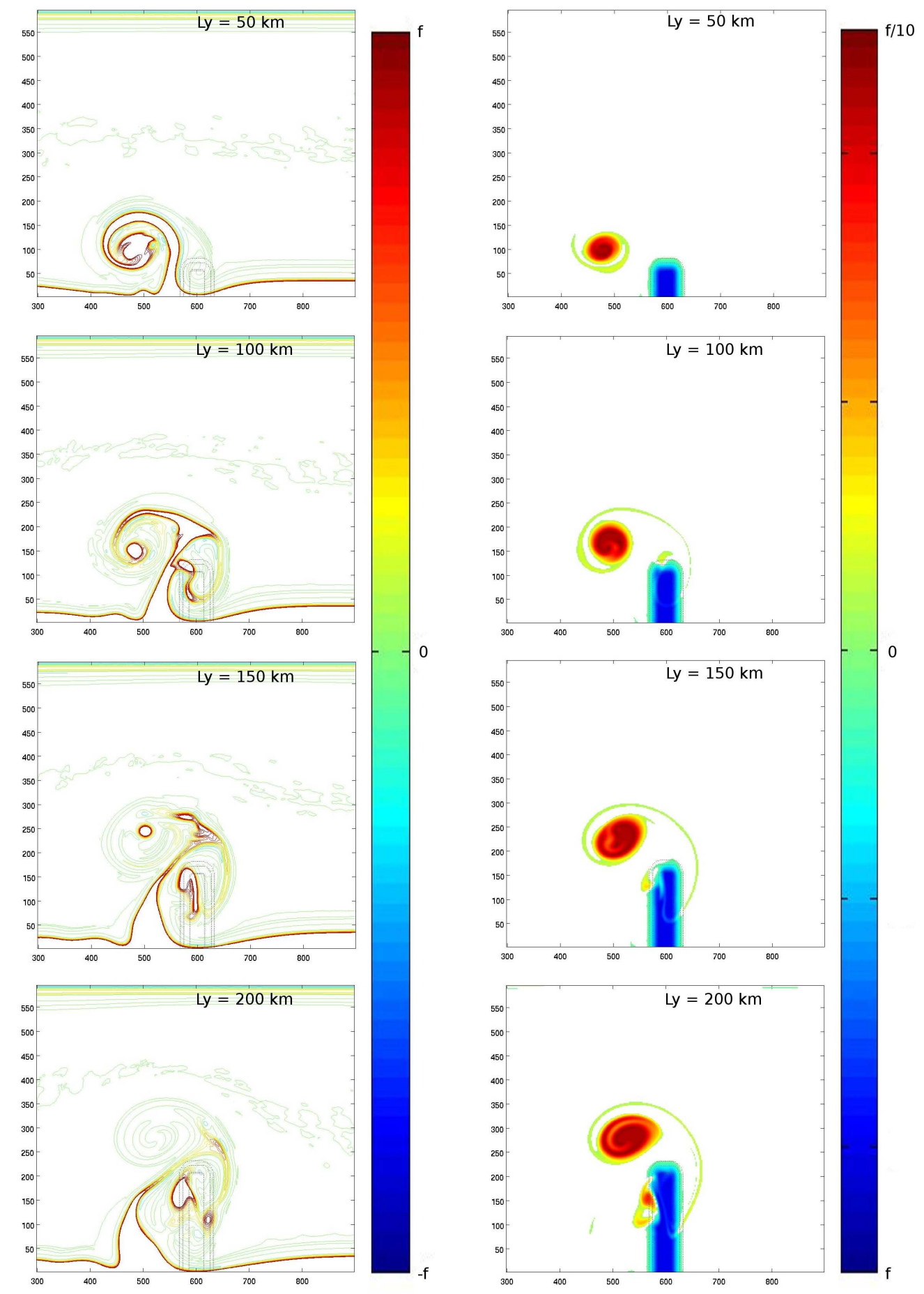

28

Figure 17: Maps of $P V A$ in the upper (left hand panel) and bottom (right hand panel) layers at $t=42$ days for the $L y=50,100,150$ and $200 \mathrm{~km}$ experiments. 


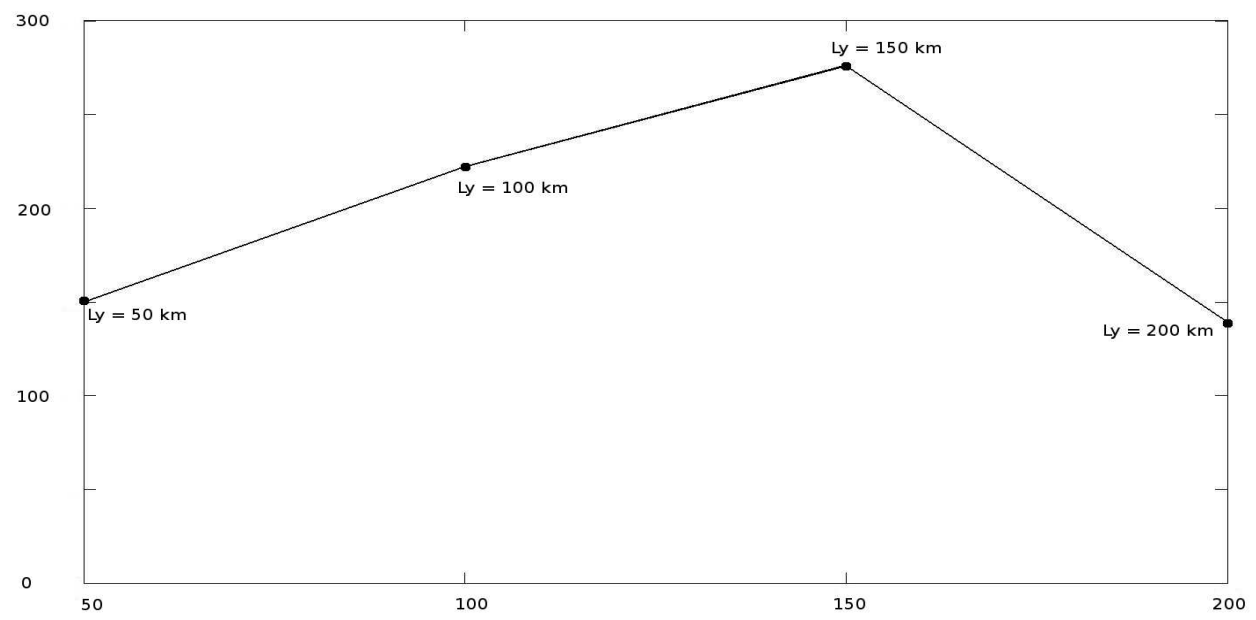

Figure 18: Offshore extent of the filament after 50 days of experiment for the $L y=50,100,150$ and $200 \mathrm{~km}$ cases cases The $\mathrm{x}$ axis is $L_{y}(\mathrm{kms})$ and the $\mathrm{y}$ axis is offshore distance $(\mathrm{kms})$.

$t=42$ days (right hand panel) for the $\mathrm{Cd}=0,310-3$, and $510^{-3} \mathrm{Nm}^{-2}$ cases. The formation and evolution of the filament in the upper layer is unchanged by the addition of the bottom friction. The effect of friction is only visible in the $P V$ of the bottom layer: As the positive $P V$ reservoir that has left the promontory to form the positive $P V A$ pole gets eroded, new higher $P V$ is generated over the promontory, resulting in weakening the negative $P V A$ pole.

As a result, bottom friction leads to a relaxation of the circulation and to a new state of rest, which allows new topographic eddies and front filamentation to occur if the wind starts blowing again.

\subsection{Influence of other topographic features}

\subsection{Capes}

Complementary experiments including a cape or a cape superimposed on a larger promontory and a canyon where performed in order to compare the impact of the coastline geometry with the topographic process proposed here. Upwelling filament dynamics have sometimes been associated with capes triggering ([Strub et al., 1991]), but most capes have large promontorylike undersea extends like Cabo Roca, Cabo Finisterre and the Estremadura promontory on the western Iberian coast.

The upper two panels in Fig. 20 show the PVA in the upper (left hand side) and bottom (right hand side) layer for two sizes of capes, both having the same Gaussian shape. Viscosity induces increased diabatic effects near boundaries which results here in the development of a layer of positive PVA. The presence of a cape allows this PVA to detach from the coast and wrap into a positive PVA pole. This process generates a cyclonic vortex downstream of the cape that in turn induces an offshore displacement of the outcropped front for small capes (cape 1 is $50 \mathrm{~km}$ long and $25 \mathrm{~km}$ wide), and in the generation of a thin filament parallel to the coast 

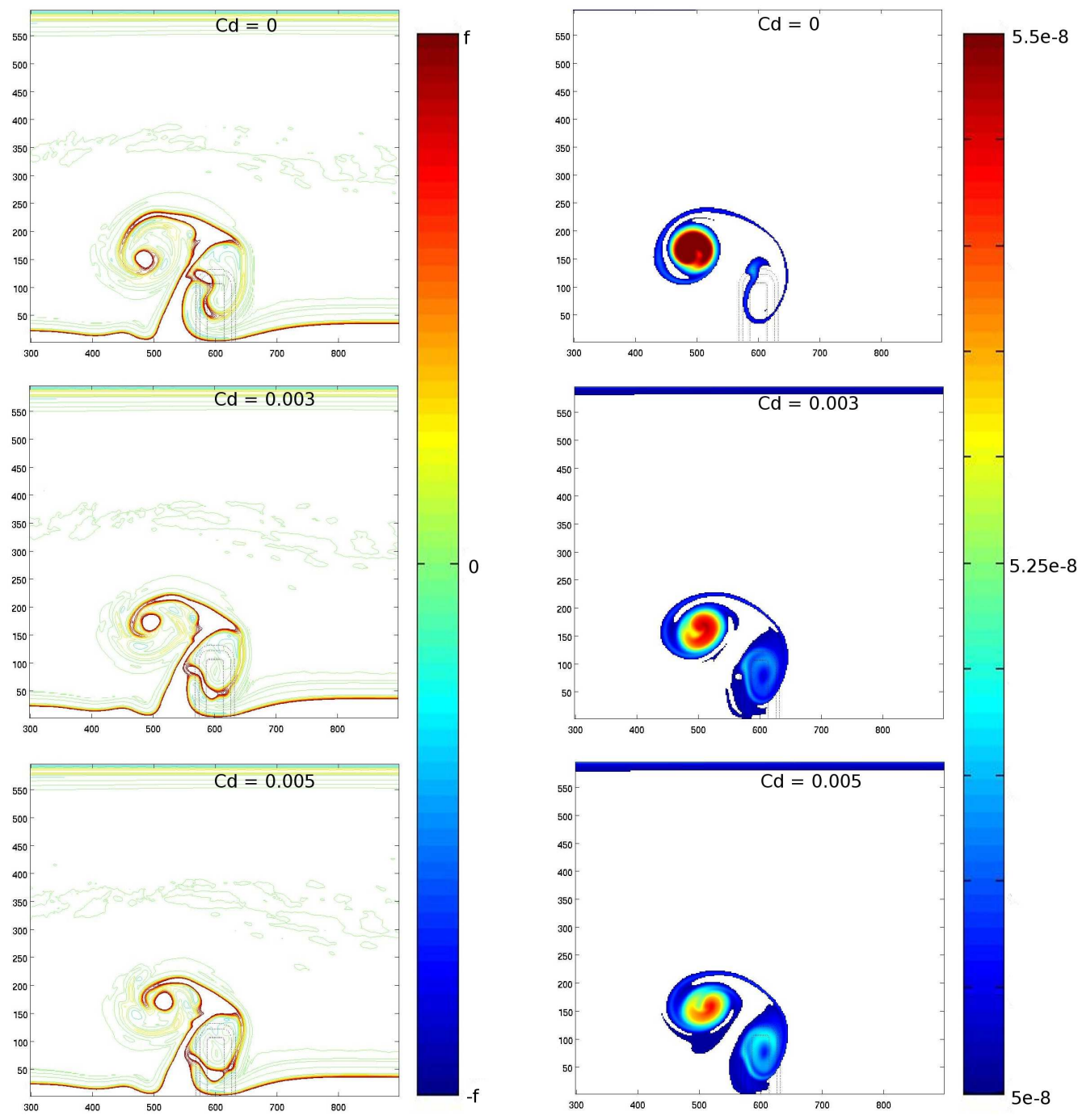
for the larger capes (cape 2 is $100 \mathrm{~km}$ long and $100 \mathrm{~km}$ wide). Superimposing the first cape with the reference promontory (third panel) leads to a long and thin filament pointing offshore and developing downstream of the cape. This combination of cape and promontory seems to be particularly efficient to generate the filament, because the cape alters the anticyclonic circulation on the promontory, so that the filament keeps on growing offshore instead of rolling up around the promontory.

\subsection{Canyons}

The lower panels in Fig. 20 show the effect of a canyon having the same shape as the reference promontory. Such a configuration generates a cyclonic circulation above the promontory and an anticyclonic one downstream. As a result, the jetlike current between both eddies is now directed shoreward and can not generate a filament. However, the cyclonic circulation induces an offshore current upstream of the canyon that, even though associated with a much weaker current than the promontory case, can advect the upwelling front offshore. This is observed on Fig. 20 where a filament forms in the upper layer upstream of the canyon. Also notice that the upwelling front disappeared downstream of the promontory. There probably also exists an optimal shape of the canyon for the development of the filament, but this is beyond the scope of the present study and we simply conclude that canyons can also play an important role in the formation of trapped and long upwelling filaments.

\subsection{Combination of canyons and promontories}

!!!???YM In many upwelling systems, the topography is not as simple as a promontory or a canyon surrounded by a large flat bottom area. The bottom topography off the Rias Baixas, North of the Iberian upwelling system is a good example of a succession of canyons and promontories. In that case, a more complex dynamics is expected. To explore the impact of such a combination on the filament development and trapping, we set up two configurations : One with a canyon downstream of the promontory and the other with a canyon upstream of the promontory. Both are tested with two values of promontory/canyon height/depth. Figure 21 shows the $P V A$ in layer 1 (left hand side panels) and in layer 2 (right hand side panels) for a $H_{t}=200 \mathrm{~m}$ promontory/canyon combination (panel 1), $H_{t}=100 \mathrm{~m}$ promontory/canyon combination (panel 2), $H_{t}=200 \mathrm{~m}$ canyon/promontory combination (panel 3)and $H_{t}=100 \mathrm{~m}$ canyon/promontory combination (panel 4). In all 4 cases, the bottom layer $P V A$ dynamics is slightly complicated because of the generation of $4 P V A$ poles, but the main effects associated with the effect of isolated topographic features are still visible : as the water initially situated over the flat bottom crosses the promontory (canyon), a negative (positive) PVA is generated, while a positive (negative) $P V A$ is generated as the water from the promontory(canyon) crosses the canyon (promontory). Another negative (positive) $P V A$ is formed when the water originally located above the canyon reaches the flat bottom area. Once the water columns originally situated upstream of the promontory (canyon) have completely crossed the topography and reached the downstream flat bottom area, the $P V A$ distribution is as follow : 2 trapped opposite sign $P V A$ over the promontory and the canyon, and two free opposite sign $P V A$ downstream of the topography, evolving as a dipole. Note that in the $H_{t}=200 \mathrm{~m}$ cases, there is a slight multipolarisation because of stronger non linear interaction between the higher $P V A$ poles, while in the $H_{t}=100 \mathrm{~m}$ case, there is a stronger advection of the free $P V A$ poles.

Even though both canyon/promontory and promontory/canyon combinations produce long filaments, there is a dynamical difference between them : In the promontory/canyon case, the most 

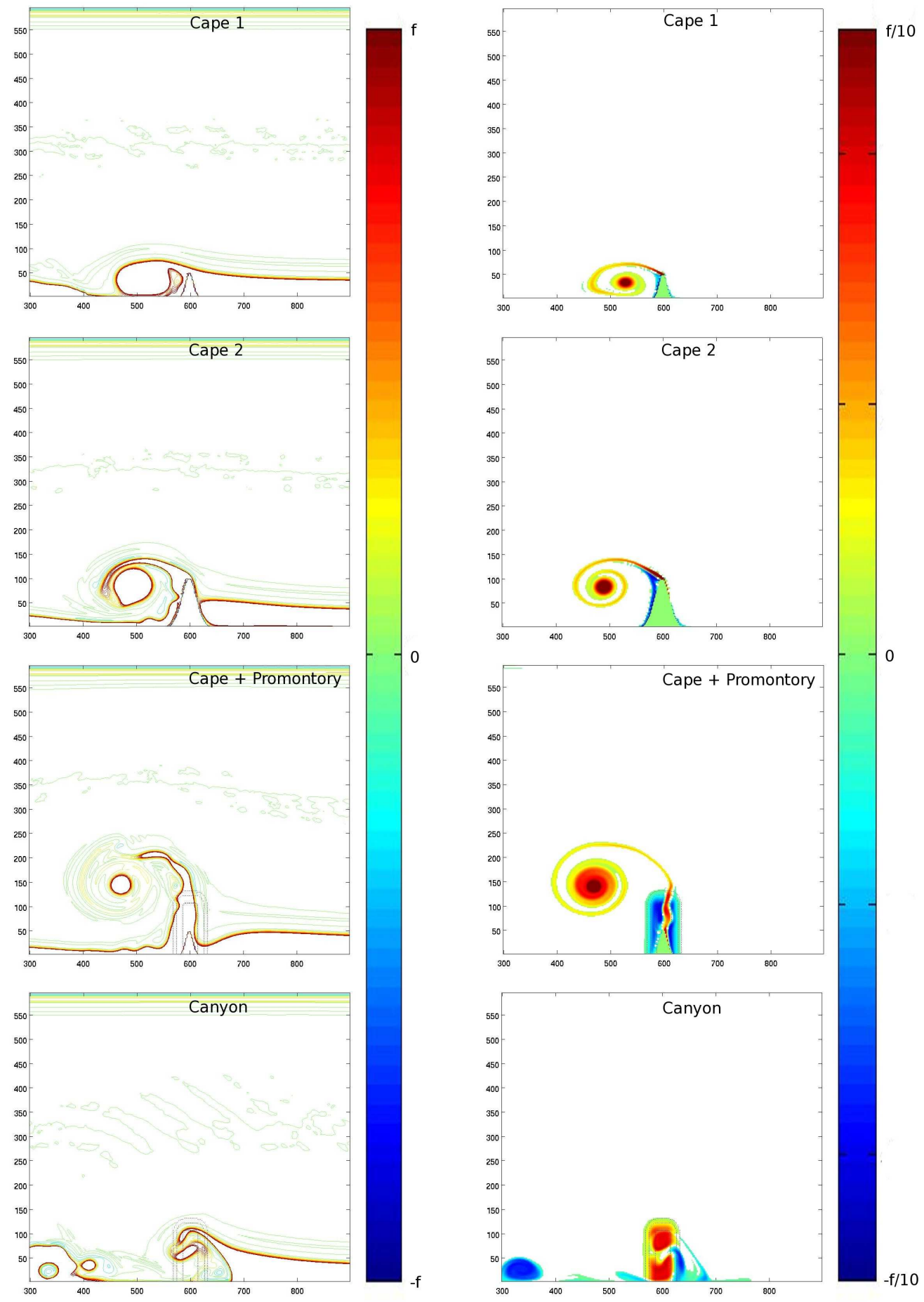

32

Figure 20: Maps of $P V A$ in the upper (left hand panel) and bottom (right hand panel) layers at $t=42$ days for the two capes experiments (panels 1 and 2), the cape over the promontory experiment (panel 3) and the canyon experiment (panel 4). 
upstream $P V A$ pole is the trapped negative one associated with the promontory which results in an offshore deviation of the front, while in the canyon/promontory combination, the trapped positive $P V A$ associated with the canyon entrain the front inshore, inhibiting a proper filamentation over the topography. This results in a major difference in the nature of the observed filaments : while the filament of the promontory/canyon combination is generated by the effect of the trapped dipole resulting in a permanently trapped filament between the promontory and the canyon, the filament generated by the canyon/promontory combination is generated by the effect of the free dipole resulting in a free filament, moving downstream as it gets advected by the barotropic current.

As shown by these simple examples, the combinations of canyons and promontories with complex shapes, as existing in nature, may lead to a more drastic selection of regions for the generation of long and trapped filaments.!!!???YM

\section{Conclusion}

\subsection{Generation of long trapped filament by topographic effect}

In this paper, we have studied the formation of long trapped upwelling filaments which are ubiquitous features in all major upwelling systems. We focused on the effect of bottom topography using an idealized two-layer configuration with a wind forcing that generates an upwelling front along a vertical coastal wall in the presence of a transverse promontory or ridge. The physical process studied is based on the formation of PVA when the upwelling current interacts with the bottom topography. At rest, in the bottom layer, the promontory is a positive potential vorticity anomaly pool, because the ambient potential vorticity is stronger over the promontory than in the rest of the domain. As the upwelling current sets up, this high potential vorticity gets advected downstream of the promontory by the alongshore current, and is replaced by lower potential vorticity water, generating a negative potential vorticity anomaly $(P V A)$ over the promontory, and a positive potential vorticity anomaly downstream. The positive $P V A$ is advected offshore by the trapped negative pole current field, and evolves into a cyclonic vortex eventually advected downstream. The negative $P V A$ induces an anticyclonic circulation anomaly that remains trapped above the topography and, if it is strong enough, will modify the mean upwelling current. This produces a geostrophic offshore flow on the downstream side of the promontory which is able to distort the upwelling front and then forms a meander. The latter finally evolves into a thin filaments that grows offshore, and that may be sometimes slightly rolling up around the topographic eddies. The barotropic part of the circulation plays the main role in the latter process.

Then, we evaluated the influence of baroclinic instability on the formation of the meanders generated by topographic effects using stable and unstable configurations. The modelled stable current is able to generate a very large and stationary filament when interacting with topography whereas baroclinic instabilities of the jet produce additional numerous shorter meanders quickly propagating downstream that have a weak influence on the topographic filament development. The characteristics of the meanders and filaments associated with baroclinic instability are sensitive to the stratification and, as found in some previous studies (see [Haidvogel et al., 1991, Strub et al., 1991]), long filament can also emerge in this case, but the trapping of the filament and their development at identical locations can only be explained by topographic effects.

We have also found that the deviation of the upwelling current by a cape and the generation of vorticity by the viscous boundary layer generate a cyclonic pool of cold water downstream of the 


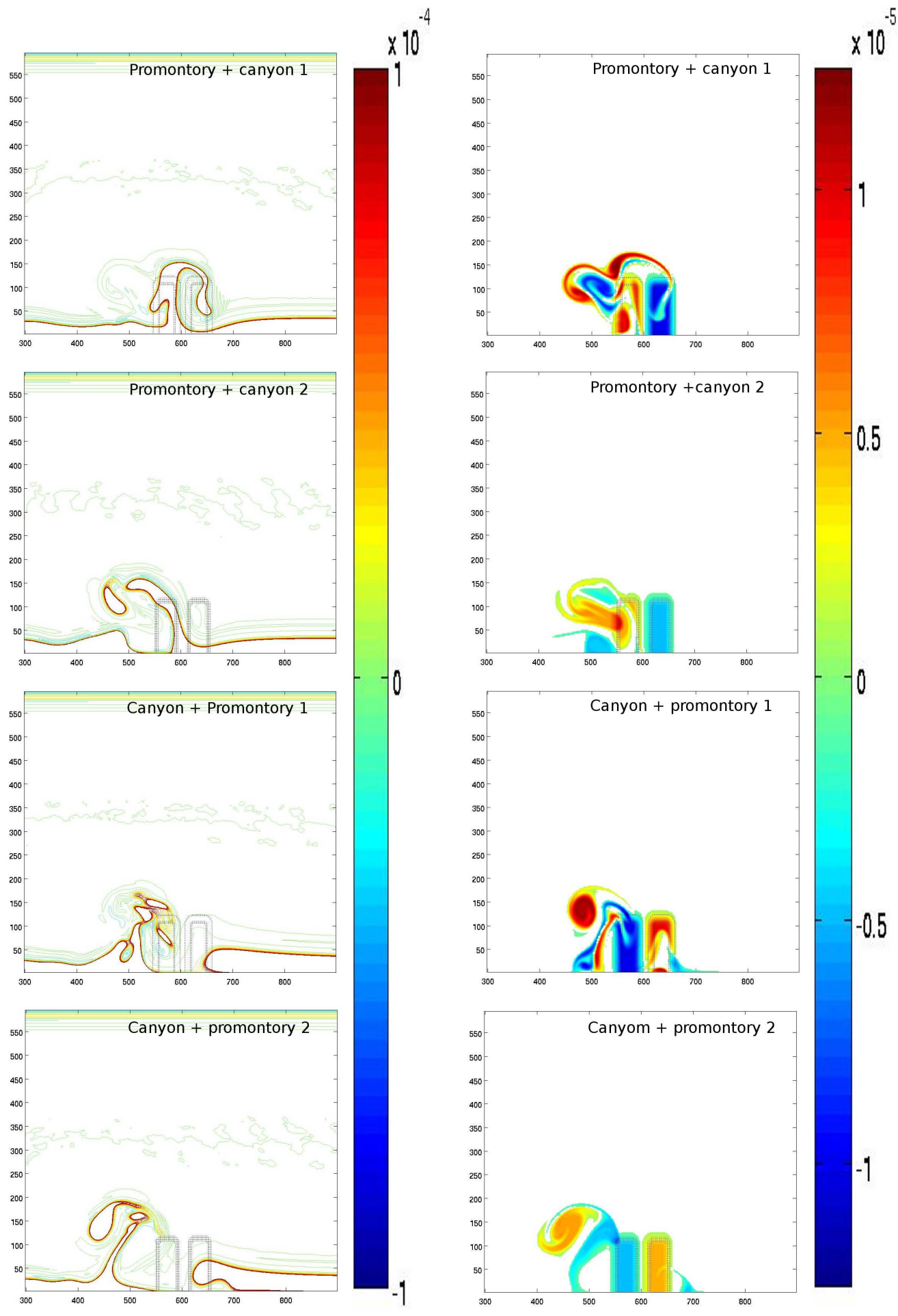

34

Figure 21: Maps of $P V A$ in layer 1 (left hand side panels) and in layer 2 (right hand side panels) for a $H_{t}=200 m$ promontory/canyon combination (panel 1), $H_{t}=100 \mathrm{~m}$ promontory/canyon combination (panel 2), $H_{t}=200 \mathrm{~m}$ canyon/promontory combination (panel 3)and $H_{t}=100 \mathrm{~m}$ canyon/promontory combination (panel 4). 
cape rather than a long offshore filament. Thus, at least for the simplified configurations used in the present paper, the process involved in the generation of upwelling filaments at capes is rather associated with the submarine promontories that generally exist in their continuity, modifying the potential vorticity structure of the current as explained above.

The topographic eddies are generated by the barotropic circulation associated with the upwelling development. Their advective effect on the upwelling front as well as the formation of filaments are again associated with their barotropic vorticity. The process thus mainly involves the barotropic circulation. As a consequence, modifying the stratification does not strongly modify the mechanism we have identified, nor the generation of topographic filaments as long as the upwelling front is formed.

Increasing the duration of the wind forcing induces a further extension of the upwelling front, and intensifies the strength of the upwelling barotropic current but not of the topographic eddies. The cyclonic eddy detaching from the topography is thus advected further downstream which, together with the change in the upwelling front extension, modify the characteristics of the filament. In our case the filament width increases and bends downstream when applying wind forcing for a longer time. Let us note that increasing the duration of the wind forcing also increases the source of diabatic $P V$ generation (see [Morel et al., 2006]) and thus the instability of the current. This was not investigated in the present paper, but we expect that, as a result, the relative importance of the unstable structure increases as the forcing lasts longer, so that a greater part of the offshore transport can be attributed to the instability of the jet in this case. This should however not change our conclusions on the trapping of long upwelling filaments.

Sensitivity tests to the shape of the topographic feature have shown that the width, height, length and slope of the topography are important parameters for the process. Multipolarisation of the $P V A$, decreasing the coherence and the length of the surface filaments, can occur in the case of a too wide or too tall promontory. The height of the promontory controls the available $P V A$ pool, so that a too small promontory can not produce a large stationary filament, but neither can a very tall promontory above which flows and $P V A$ can barely form. There exists optimal values of the topography characteristics to maximize the offshore extension of the filament. In other words, the formation of long trapped upwelling filaments by topographic features is a selective process and is restricted to some height, width, slope and length ranges.

\subsection{Discussion and perspectives}

!!!???YM Even though our model is of the simplest, it allows to clearly identify a dynamical process which seems relevant to explain the stationarity and the repeatability of upwelling filaments at some particular locations. This simplicity however makes a thorough comparison with observed current structures a difficult task, but a qualitative discussion on some general patterns remains possible.

It is important to note that the various upwelling systems develop over very different topographies : if the Iberian or the Chilean margins show complicated patterns with a succession of ridges and canyons in the continuation of the rias, the North African topography is a combination of a smooth margin with a few well defined promontories (Cape Ghir, Cape Blanc). As shown in section 4.4 and 4.9, the generation of trapped filaments is highly sensitive to the topographic configuration, and the multiplicity and greater variability of upwelling filaments along the WestIberian shelf could be explained by this more complex bottom topography. If the isolated Cape Ghir promontory is a scholar case for the generation of well defined trapped anticyclone, triggering a large long lived stationary filament, the multiple $P V A$ poles generated over the complex Iberian topography must lead to non-linear interactions, including fragmentation and merger of 
the PVA poles leading to the observed shorter-lived, less coherent filament with higher spatial and temporal variability.

???YM ATTENTION, je pense que les conditions atmospheriques peuvent aussi pas mal jouer : les vents sur la faade iberique sont assez inconstants et expliquent sans doute aussi pas mal la variabilit observes. ???YM

Note also that the present study does not exclude the processes invoked by previous studies to explain the generation of upwelling filaments. Indeed, topographically trapped eddies can combine or interact with other filamentation processes. The Southern North-West African upwelling system, offshore of Mauritania is a good example of the multiplicity of dynamics of the upwelling filaments : Recent satellite and in-situ observations (SOLAS-ICON cruise, unpublished yet) showed a whole range of filaments with different sizes, shapes and behaviour. The intrinsic instability of the upwelling front generates pinched off meanders, developing and propagating all along the front between the Arguin's bank and cape Verde whereas a quasi permanent filament associated with an anticyclonic eddy is found over the Cape Blanc promontory. Even though this filament is quasi-permanently rooted over the Cap Blanc promontory, its offshore extension shows a high spatial and temporal variability. This variability is believed to result from interactions with an external mesoscale turbulence field resulting from the presence of the baroclinically unstable Cape Verde front in the vicinity of the Mauritanian upwelling system. This confirms the idea of [Strub et al., 1991] that filaments could result of the combined effect of various processes like jet instability and the interaction with an offshore eddy field. The role of bottom topography is finally added to justify the stationarity and repeatability of filaments. ???YM ATTENTION: je trouve que ce dernier paragraphe brouille le message. Il faut que le lecteur reparte avec comme ide claire que jusqu' prsent seul le mcanisme propos ici permet d'expliquer la prsence de long filament pigs se dveloppant systmatiquement au mme endroit. D'autres mcanismes peuvent soit gnrer des filaments (mais a priori ayant des caractristiques en terme de stationnarit/pigeage diffrent) soit interagir avec le prsent effet pour en diminuer ou en augmenter les effets. Je suggre de rcrire ce paragraphe sur la base de ce fil conducteur. Tel quel ce paragraphe remet tout zro mon sens : on comprend que finalement d'autre mcanisme peuvent trs bien expliquer le mme comportement sans qu'on donne d'explication cela. ???YM

\section{!!!???YM}

The two-layer adiabatic model we have used is however very simplified and in nature, many other parameters and many additional processes, that have not been studied here, can influence the development of topographic eddies and formation of upwelling filaments, among which :

- the existence of an extended shelf and a continental slope ;

- the existence of a more complex and realistic large scale circulation (presence of a deep poleward undercurrent) associated with or preceding the upwelling development;

- the influence of a more complicated stratification and of the specific dynamics of the mixed layer or the influence of mixing in general;

- the influence of the planetary $\beta$ effect;

- the influence of bottom friction.

The beta effect seems of particular importance as it strongly influences the dynamics of eddies and induces westward propagation. We can thus imagine, for Eastern boundary upwellings, that any localized source of vortices such as bottom topography, but also capes, could favor the 
offshore development of filaments : indeed the cyclonic vortex developing downstream of a cape would be advected westward entraining upwelled cold waters and forming a localized filament instead of a circular patch as observed in the present study.

Another interesting subject is the influence of bottom friction. We have found here that its influence was weak, but this was expected since we considered a deep ocean. Bottom friction would obviously plays a stronger role than observed here for upwelling developing above shallow areas (corresponding to extended shelves). As its effect is to reduce the currents in the bottom layers, we expect this would limit the strength of the upwelling barotropic velocity and downstream transport.However, meanwhile it would also renew the reservoir of positive PVA above the promontory. As a result, after a period of relaxation of the winds, despite the fact that the initial positive PVA moved away from the topography, the same mechanism could be repeated for a new upwelling event.

However, even though these processes can have a strong influences on the result and their effect is worth investigating, we believe that they would not substantially modify our main result : the bottom topography plays a key role on the formation of the long upwelling filaments, whatever the direction of the current and details of the topography, $P V A$ has to form when the flow passes over topography, developing a trapped circulation whose signature extends over the entire water column and influences the upper layer dynamics advecting water parcels offshore.

Finally, more in-situ observations of early stage development of upwelling filaments are needed to possibly confirm our results and the main influence of the barotropic circulation associated with topographic eddies. This implies an extended mapping of the circulation, !!! including the deep mesoscale features which are often neglected during the mesoscale surveys because of the difficulty to sample at great depths without loosing time and thus synopticity. !!!.

\section{Acknowledgements}

Part of this study has been conducted in the frame of the MOUTON project funded by DGA (PEA 012401) and the CAIBEX project (CTM2007-66408-C02/MAR) funded by the Spanish National government. T.M. thanks Pr E.D. Barton for the useful discussions and the calculation time supplied at IIM-CSIC (Spain). V.R. is supported by a PhD grant from DGA. Satellite images where provided by Joel Sudre at LEGOS. 
References

[Alvarez-Salgado et al., 2001] Alvarez-Salgado, X.A., M.D. Doval, A.V. Borges, I. Joint, M. Frankignoulle, E.M.S Woodward, F.G. Figueiras, Off-shelf fluxes of labile materials by an upwelling filament in the NW Iberian Upwelling System. Progress in Oceanography, 51, 321-337, 2001.

[Alvarez-Salgado et al., 2007] 1varez-Salgado, X.A., Arstegui, J., Barton, E.D., Hansell, D.A., Contribution of upwelling filaments to offshore carbon export in the subtropical Northeast Atlantic Ocean. Limnology and Oceanography, 52, 1287-1292, 2007.

[Bang and Andrews, 1974] Bang, N.D., and Andrews, W.R.H., Direct current measurements of a shelf-edge frontal jet in the southern Benguela system, Journal of Marine Research, 32, 407-421, 1974.

[Barth, 1989 a] Barth, J.A. Stability of a coastal upwelling front 1. Model developing and a stability theorem, Journal of Geophysical Research, Vol. 94, 10844-10856, 1989.

[Barth, 1989 b] Barth, J.A. Stability of a coastal upwelling front 2. Model results and comparison with observations, Journal of Geophysical Research, Vol. 94, 10857-10883., 1989.

[Barth, 1994] Barth, J.A. Short-wavelength instabilities on coastal jets and fronts, Journal of Geophysical Research, Vol. 98, 16095-16115, 1994.

[Barton, 2001] Barton, E.D., M.E Inall, T,J, Sherwin and R. Torres., Vertical structure, turbulent mixing and fluxes during Lagrangian observations of an upwelling filament system off Northwest Iberia, Progress in Oceanography, Vol. 51, 249-267, 2001

[Barton, 2004] Barton, E.D., Aristegui, J., Tett, T. and Navarro-Pérrez, E., Variability in the Canary Islands Area of Filament-Eddy Exchanges, Progress in Oceanography, Vol. 62, 71-94, 2004.

[Batteen, 1997] Batteen, M.L., Wind-forced modeling studies of currents, meanders, and eddies in the California Current system Journal of Geophysical Research-Oceans, Vol 102, C1, 985-1010, 1997.

[Batteen et al., 2007] Batteen, M.L., Martinho, A.S., Miller, H.A. and McClean, J.L., A Process-Oriented Study of the Coastal Canary and Iberian Current System, Ocean Modelling, vol. 18, 1-36, 2007.

[Bleck and Boudra, 1986] Bleck., R. and Boundra, D., Wind driven spin-up in eddy-resolving ocean models formulated in isopycnic and isobaric corrdinates, Journal of Geophysical Research, vol. 91, 7611-7621, 1986.

[Bleck and Smith, 1990] Bleck., R. and Smith, L., A wind driven isopycnic coordinate model of the North and equatorial Atlantic Ocean: Model development and supporting experiments Journal of Geophysical Research, vol. 95, 32733285, 1990.

[Bleck et al., 1992] Bleck., R., Rooth, C., Hu, D., and Smith, L., 1992. Salinity driven thermocline transients in a wind and thermocline forced isopycnic coordinate model of the North Atlantic. Journal of Physical Oceanography, vol. $22,1486-1505$

[Brink, 1983] Brink, K.H., The near-surface dynamics of coastal upwelling, Progress in Oceanography, vol. 12, $223-$ $257,1983$.

[Bretherton, 1966] Bretherton, F.P., Critical layer instability in baroclinic flows, The Quarterly Journal of the Royal Meteorological Society, vol. 92, 325-334, 1966.

[Capet and Carton, 2004] Capet, X.J. and Carton, X.J. Nonlinear Regimes of Baroclinic Boundary Currents, Journal of Physical Oceanography, vol. 34, 14001409, 2004.

[Charney and Stern, 1962] Charney, J.G. and Stern, M.E., On the Stability of Internal Baroclinic Jets in a Rotating Atmosphere, Journal of the Atmospheric Sciences, vol. 19, 159172, 1962.

[Dewey et al., 1991] Dewey, R.K., Moum, J.N., Paulson, C.A., Caldwell, D.R. and Pierce, S.D., Structure and Dynamics of a Coastal Filament, Journal of Geophysical Research, vol. 96, 14885-14907, 1991.

[Flament et al., 1985] Flament, P., Armi, L. and Washburn, L., The Evolving Structure of an Upwelling Filament, Journal of Geophysical Research, vol. 90, 11765-11778, 1985.

[Garvine, 1971] Garvine, R.W., A simple model of coastal upwelling dynamics, Journal of Physical Oceanography, 1, $169-179,1971$

[Garvine, 1973] Garvine, R.W., The effects of bahtymetry on the coastal upwelling of homogeneous water, Journal of Physical Oceanography, 3, 47-56, 1973.

[Gill and Clarke, 1974] Gill, A.E. and A.J., Clarke, Wind-induced upwelling, coastal currents and sea level changes, Deep-Sea Research, vol. 21, 325-345, 1974.

[Haidvogel et al., 1991] Haidvogel, D.B., Beckmann, A. and HedStrm, K.S., Dynamical Simulations of Filament Formation and Evolution in the Coastal Transition Zone, Journal of Geophysical Research, vol. 96, 15017-15040, 1991.

[Haynes et al., 1993] Haynes, R., Barton, E.D. and Pilling, I. Development, Persistence, and Variability of Upwelling Filaments, Journal of Geophysical Research, vol. 98, 22681-22692, 1993.

[Herbette et al., 2003] Herbette, S., Morel, Y.G. and Arhan, M., Erosion of a surface vortex by a seamount, Journal of Physical Oceanography, vol. 33, (8):1664-1679, 2003. 
[Herbette et al., 2005] Herbette, S., Morel, Y.G. and Arhan, M., Erosion of a surface vortex by a seamount on the beta plane, Journal of Physical Oceanography, vol. 35, (11):2012-2030, 2005.

[Hoskins et al., 1985] Hoskins, B., McIntyre, M. and W. Robertson, On the use and significance of isentropic potential vorticity maps, Quarterly Journal of the Royal Meteorological Society, vol. 111, 877-946, 1985.

[Ikeda, 1981] Ikeda, M., Meanders and Detached Eddies of a Strong Eastward-Flowing Jet Using a Two-Layer QuaiGeostrophic Model, Journal of Physical Oceanography, vol. 11, 526-540, 1981.

[Ikeda, 1989] Ikeda, M., Lygre, K. and Sandven, S., A Process Study of Mesoscale Meanders and Eddies in the Norwegian Coastal Current, Journal of Physical Oceanography, vol. 19, 20-35 1989.

[Killworth, 1980] Killworth, P.D., Barotropic and baroclinic instability in rotating stratified fluids, Dynamics of Atmosphere and Ocean, vol. 4, 143-184, 1980.

[Kostianoy and Zatsepin, 1996] Kostianoy, A.G. and Zatsepin, A.G., The West African coastal upwelling filaments and cross-frontal water exchange conditioned by them, Journal of Marine Systems, vol. 7, 349-359, 1996.

[Lentz and Chapman, 2004] Lentz, S.J. and D.C., Chapman, The importance of nonlinear cross-shelf momentum flux during wind-driven coastal upwelling, Journal of Physical Oceanography, 34, 2444-2457, 2004.

[Marchesiello et al., 2003] . Marchesiello, P., J. C. McWilliams and A. Shchepetkin, Equilibrium structure and dynamics of the California Current System. J. Phys. Oceanogr., 33, 753-783, 2003.

[McIntyre and Norton, 1990] McIntyre, M., and W. Norton, Dissipative wave-mean interactions and the transport of vorticity or potential vorticity, Journal of Fluid Mechanics, vol. 212, 403-435, 1990.

[McWilliams and Gent, 1980] McWilliams, J.C., and Gent, P.R., 1980. Intermediate models of planetary circulations in the atmosphere and the ocean. Journal of Atmospherical Sciences, vol. 37, 1657-1678.

[Morel and McWilliams, 2001] Morel, Y. and McWilliams, J., Effects of Isopycnal and Diapycnal Mixing on the Stability of Oceanic Currents, Journal of Physical Oceanography, vol. 31, 2280-2296, 2001.

[Morel et al., 2006] Morel, Yves, G., Darr, D. S. and C. Talandier, Possible sources driving the Potential Vorticity structure and long-wave instability of coastal upwelling and downwelling currents Journal of Physical Oceanography, vol. 36, 875-896, 2006

[Morel and Thomas, 2009] Morel, Y. and Thomas, L.N., Ekman drift and vortical structures, Ocean modelling, vol. 27, 185-197, 2009.

[Navarro-Pérrez and Barton, 1998] Navarro-Pérrez, E. and Barton, E.D., The Physical Structure of an Upwelling Filament off the North-West African Coast during August 1993, South African Journal of Marine Science, vol. 19, 61-73, 1998

[O'Brien and Hurlburt, 1972] O'Brien, J.J. and Hurlburt, H.E., A numerical model of coastal upwelling, Journal of Physical Oceanography, vol. 2, 1972.

[Pringle, 2002] Pringle, J.M., Enhancement of wind-driven Upwelling and Downwelling by alongshore bathymetric variability, Journal of Physical Oceanography, 32, 31013112, 2002.

[Relvas et al., 2007] Relvas, P., Barton, E.D., Dubert, J., Oliveira, P.B., Peliz, A., da Silva, J.C.B. and A.M.P. Santos, Physical oceanography of the western Iberia ecosystem: Latest views and challenges, Progress in Oceanography, 74, 149-173, 2007.

[Rossi et al., 2009] Rossi, V., Morel, Y. and Garcon, V., Effect of the wind on the shelf dynamics: Formation of a secondary upwelling along the continental margin, Ocean Modelling, 2009, doi : 10.1016/j.ocemod.2009.10.002.

[Roed and Shi, 1999] Roed, L. P., and X. B. Shi, A numerical study of the dynamics and energetics of cool filaments, jets and eddies off the Iberian Peninsula. Journal of Geophysical Research, 104(C12), 29817-29841, 1999.

[Sanchez et al., 2008] R. F. Sanchez, P. Relvas, A. Martinho, and P. Miller, Physical description of an upwelling filament west of Cape St. Vincent in late October 2004, Journal of Geophysical Research, Vol. 113, C07044, doi:10.1029/2007JC004430, 2008.

[Shi and Roed, 1999] X. B. Shi and Roed, L. P., Frontal Instabilities in a Two-Layer, Primitive Equation Ocean Model, Journal of Physical Oceanography, Vol. 29, 948-968, 1999.

[Stern and Chassignet, 2000] M.E. Stern and E.P. Chassignet, Mechanism of eddy separation from coastal currents , Journal of Marine Research, Vol. 58, 269-295, 2000.

[Strub et al., 1991] Strub, T.P., Kosro, P.M., and Huyer, A., The nature of the cold filaments in the California Current System, Journal of Geophysical Research, vol. 96, 14743-14768, 1991.

[Thomas, 2005] Thomas, L.N., Destruction of potential vorticity by winds, J. Phys. Ocean., vol. 35, 2457-2466, 2005.

[Verron and Le Provost, 1985] Verron. J. and Le Provost, C., A numerical study of quasi-geostrophic flow over topography, J. Fluid Mech., vol. 154, 231-252, 1985.

[Viera and Grimshaw, 1994] Viera, F. and Grimshaw, R., Topographic Forcing of Coastal Mesoscale Phenomena: Filamentation, Vortex Formation, and Eddy Detachment, Journal of Physical Oceanography, vol. 24, 1433-1448, 1994.

[Washburn and Armi, 1988] Washburn, L. and Armi, L., Observations of Frontal Instabilities on an Upwelling Filament, Journal of Physical Oceanography, vol. 18, 1075-1092, 1988.

[Winther et al., 2007] Winther, N.G., Morel, Y.G. and Evensen, G., Efficiency of high order numerical schemes for 
momentum advection, Journal of Marine Systems, vol. 67 (1-2), 31-46, 2007. 


\begin{tabular}{|c|c|c|c|c|c|c|c|c|c|}
\hline$\delta t_{t}$ & $\delta t_{c}$ & $\delta x$ & $N_{x}$ & $N_{y}$ & $N_{\text {days }}$ & $v$ & $T_{x}$ & $f$ & $H_{2}+H_{1}$ \\
\hline \hline $5 \mathrm{~s}$ & $200 \mathrm{~s}$ & $2000 \mathrm{~m}$ & $601 \mathrm{pts}$ & $301 \mathrm{pts}$ & 50 days & $0.3 \mathrm{~m}^{2} \mathrm{~s}^{-1}$ & $-0.2 \mathrm{Nm}^{-2}$ & $10^{-4} \mathrm{~s}^{-1}$ & $2050 \mathrm{~m}$ \\
\hline
\end{tabular}

Table 1: Table of the model parameters kept fixed in all experiments.

\begin{tabular}{|c|c|c|c|c|c|c|c|c|}
\hline Experiment & $d \rho\left(\mathrm{kgm}^{-3}\right)$ & $C d$ & $H_{1}(\mathrm{~m})$ & Forcing duration (days) & $H_{t}(\mathrm{~m})$ & $L_{x}(\mathrm{~km})$ & $L_{y}(\mathrm{~km})$ & $d L(\mathrm{~km})$ \\
\hline Ref & 1 & 0 & 50 & 10 & 200 & 20 & 100 & 20 \\
\hline Inst & 1 & 0 & 50 & 10 & 200 & 20 & 100 & 20 \\
\hline Notopo frc 10 & 1 & 0 & 50 & 10 & $\underline{0}$ & $\underline{0}$ & $\underline{0}$ & $\underline{0}$ \\
\hline Notopo frc 20 & 1 & 0 & 50 & 20 & $\underline{0}$ & $\underline{0}$ & $\underline{0}$ & $\underline{0}$ \\
\hline Notopo frc 30 & 1 & 0 & 50 & $\underline{30}$ & $\underline{0}$ & $\underline{0}$ & $\underline{0}$ & $\underline{0}$ \\
\hline Notopo frc 40 & 1 & 0 & 50 & $\underline{40}$ & $\underline{0}$ & $\underline{0}$ & $\underline{0}$ & $\underline{0}$ \\
\hline $\mathrm{Cd} 3 \mathrm{e}-3$ & 1 & $\underline{3.10^{-3}}$ & 50 & $\overline{10}$ & 200 & 20 & 100 & $\overline{20}$ \\
\hline $\mathrm{Cd} 5 \mathrm{e}-3$ & 1 & $5.10^{-3}$ & 50 & 10 & 200 & 20 & 100 & 20 \\
\hline frc time 20 & 1 & 0 & 50 & 20 & 200 & 20 & 100 & 20 \\
\hline frc time 30 & 1 & 0 & 50 & 30 & 200 & 20 & 100 & 20 \\
\hline frc time 40 & 1 & 0 & 50 & 40 & 200 & 20 & 100 & 20 \\
\hline$H_{t} 50$ & 1 & 0 & 50 & 10 & $\underline{50}$ & 20 & 100 & 20 \\
\hline$H_{t} 100$ & 1 & 0 & 50 & 10 & $\underline{\overline{100}}$ & 20 & 100 & 20 \\
\hline$H_{t} 300$ & 1 & 0 & 50 & 10 & 300 & 20 & 100 & 20 \\
\hline$H_{t} 500$ & 1 & 0 & 50 & 10 & $\underline{500}$ & 20 & 100 & 20 \\
\hline$H_{t} 1000$ & 1 & 0 & 50 & 10 & 1000 & 20 & 100 & 20 \\
\hline$H_{t} 1500$ & 1 & 0 & 50 & 10 & 1500 & 20 & 100 & 20 \\
\hline$L_{x} 0$ & 1 & 0 & 50 & 10 & 200 & $\underline{0}$ & 100 & 20 \\
\hline$L_{x} 50$ & 1 & 0 & 50 & 10 & 200 & $\underline{50}$ & 100 & 20 \\
\hline$L_{x} 100$ & 1 & 0 & 50 & 10 & 200 & 100 & 100 & 20 \\
\hline$L_{y} 50$ & 1 & 0 & 50 & 10 & 200 & 20 & $\underline{50}$ & 20 \\
\hline$L_{y} 150$ & 1 & 0 & 50 & 10 & 200 & 20 & 150 & 20 \\
\hline$L_{y} 200$ & 1 & 0 & 50 & 10 & 200 & 20 & $\underline{200}$ & 20 \\
\hline $\mathrm{dL} 0$ & 1 & 0 & 50 & 10 & 200 & 20 & 100 & $\underline{0}$ \\
\hline dL 10 & 1 & 0 & 50 & 10 & 200 & 20 & 100 & 10 \\
\hline $\mathrm{dL} 40$ & 1 & 0 & 50 & 10 & 200 & 20 & 100 & $\underline{40}$ \\
\hline$H_{1} 25$ & 1 & 0 & 25 & 10 & 200 & 20 & 100 & 20 \\
\hline$H_{1} 100$ & 1 & 0 & 100 & 10 & 200 & 20 & 100 & 20 \\
\hline$H_{1} 200$ & 1 & 0 & $\underline{200}$ & 10 & 200 & 20 & 100 & 20 \\
\hline$d \rho 0.5$ & 0.5 & 0 & 50 & 10 & 200 & 20 & 100 & 20 \\
\hline$d \rho 2$ & 2 & 0 & 50 & 10 & 200 & 20 & 100 & 20 \\
\hline$d \rho 3$ & 3 & 0 & 50 & 10 & 200 & 20 & 100 & 20 \\
\hline
\end{tabular}

Table 2: Table of the model parameters that were varied in the various experiments. 